\title{
Hunting in Unknown Environments with Dynamic Deforming Obstacles by Swarm Robots
}

\author{
Hongqiang Zhang ${ }^{1,2}$, Jing Zhang ${ }^{1}$, Shaowu Zhou ${ }^{2}$, Puren Ouyang ${ }^{3,4}$ \\ and Lianghong $\mathrm{Wu}^{2}$ \\ ${ }^{1}$ College of Electrical and Information Engineering, Hunan University, Changsha, \\ Hunan, 410082, China \\ ${ }^{2}$ College of Information and Electrical Engineering, Hunan University of \\ Science and Technology, Xiangtan, Hunan, 411201, China \\ ${ }^{3}$ Department of Aerospace Engineering, Ryerson University, Toronto, Ontario, \\ M5B2K3, Canada \\ ${ }^{4}$ College of Mechanical and Electrical Engineering, Hunan University of \\ Science and Technology, Xiangtan, Hunan, 411201, China \\ hongniuok@qq.com ${ }^{1,2}$,zhangj@hnu.cn ${ }^{1}$,shaowuzhou@163.com ${ }^{2}$, \\ pouyang@ryerson.ca ${ }^{3,4}$, lhwu@hnust.edu.cn ${ }^{2}$
}

\begin{abstract}
In this paper, a self-organizing method, which is based on a simplified virtual-force model, is proposed for nonholonomic mobile swarm robots hunting in unknown environments. First, the motion models for the hunted target in unknown cluttered environments are designed. Next, through the decomposition of hunting behaviors under cluttered environments, a simplified virtual-force model is formed. Then, based on the virtual-force model, a control method is designed for swarm robots following motions of barriers and the target. The method only needs the location information of the target and two nearest neighbors, so it is easy to be calculated and realized. After that, the stability of the hunting system is analyzed and the control parameter ranges are achieved. Simulation results for different situations and comparative analyses demonstrate that the proposed hunting method has good performance of obstacles avoidance and flexibility.
\end{abstract}

Keywords: Wheeled robots, nonholonomic motion planning, distributed robot systems, swarm robots, hunting

\section{Introduction}

Swarm robotics is an approach to collective robotics, inspired from the self-organized behaviors of social animals. Through simple rules and local interactions, swarm robotics aims at designing robust, scalable, and flexible collective behaviors for the coordination of large numbers of robots [1]. Even if a number of independent single robots that are equipped with a sophisticated hardware and software configuration may not perform some tasks, which may be achieved by swarm robots [2-8].

There are many works dealing with cooperative hunting of robots [9-12]. In [13-23], hunting algorithms are mainly based on artificial physics (AP) $[15,22]$, behavior-based (BB)[16], potential functions (PF) [16, 18], and rules based (RB) $[17,19,21]$.The discussion in these references is mainly for systems with few robots, using advanced communication 
among robots, and the control algorithm require the information of all the other robots and obstacles. Since great communication capacity needed, less fault tolerance, difficult to expand

the robots number, these control methods are not suitable for swarm robots hunting control.

In recent years, related research has been reported by scholars on a group of robots to round up [24-29]. There are few strategies to effectively use swarm robots system itself to hunt, especially in unknown complex environments with all kinds of obstacles. Huang et al. [30] proposed a self-organizing cooperative hunting approach by swarm robotic systems based on RB, i.e., a loose-preference rule (LP-rule), which is the most simple and efficient method of hunting presently. However, the rule is complex and there is no obstacle in the hunting environment.

To the best of our knowledge, the problem of hunting in moving and deforming obstacles environments by using a swarm of robots has never been investigated so far. As we know, robots having their own sensors and effectors can make use of any or partial AP force, which they can perceive [31]. Since AP bears some similarity with RB approach, can we place the $\mathrm{RB}$ approach in hunting on a firmer physics foundation and to make more in-depth analyses and understanding of the characteristics of self-organization hunting and the emergent of swarm intelligence? Furthermore, can we just use the position information of the target and the two nearest neighbors to accomplish self-organization hunting and get a more systematic parameters set?

In response to these challenges, according to the characteristics of animal self-organizing motions in cooperative hunting in unknown environments, a simplified virtual-force model based on AP methodology is formed, which only requires the positions information of the target and the two nearest neighbors (including robots, dynamic deforming obstacles, static non-convex, and convex obstacles). The simulations results under different conditions show that the hunting method based on the simplified virtual-force model is feasible. Its stability analyses are advantageous to the system parameter setting and it is prone to obtain oscillation small and the better trajectory of hunting process.

The remainder of this paper is organized as follows: In section 2, description of swarm robots motion model and self-organization hunting tasks are introduced. Section 3 presents the self-organization algorithm for cooperative hunting, followed by stability analysis of the self-organization algorithm in Section 4. The simulation experiments in various environments with unknown dynamic deforming obstacles are given in Section 5, followed by contrast analysis in Section 6. Finally, conclusions are presented in Section 7.

\section{Model formation}

To realize cooperative hunting, individual kinematics equations, related functions, and hunting task model are given in this section.

\subsection{Robots Motion Model and Related Functions}

Considering a swarm of $m$ identical wheeled mobile robots, assume that each robot has a structure, as shown in Figure 1. The kinematics equations of robot $h_{j}$ with pure rolling and non-slipping are expressed as follows:

$$
\left\{\begin{array}{l}
\dot{x}_{j}(t)=v_{j}(t) \cos \theta_{j}(t) \\
\dot{y}_{j}(t)=v_{j}(t) \sin \theta_{j}(t) \\
\dot{\theta}_{j}(t)=\omega_{j}(t)
\end{array}\right.
$$


where $v_{j}(t)$ and $\omega_{j}(t)$ are the linear velocity and angle velocity of $h_{j}$, respectively, and satisfy $\left|v_{j}(t)\right| \leq \mathrm{v}_{\mathrm{m}}^{H},\left|\omega_{j}(t)\right| \leq \omega_{\mathrm{m}}^{H}$. Linear acceleration and angle acceleration satisfy $\left|\dot{v}_{j}(t)\right| \leq \mathrm{a}_{\mathrm{m}}^{H}, \quad\left|\dot{\omega}_{j}(t)\right| \leq \omega_{\mathrm{am}}^{H}$, where $\mathrm{v}_{\mathrm{m}}^{H}$ and $\mathrm{a}_{\mathrm{m}}^{H}$ are the maximum linear velocity and the maximum linear acceleration, respectively; $\omega_{\mathrm{m}}^{H}$ and $\omega_{\mathrm{am}}^{H}$ are the maximum angle velocity and the maximum angle acceleration, respectively.

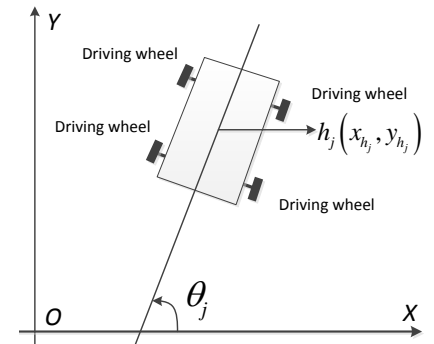

Figure 1. Schematic Model for the Wheeled Mobile Robot $h_{j}$

The applying force functions of the target and objects (includes robots, static or dynamic obstacles) during the process of hunting are given as follows:

$$
\begin{gathered}
f_{t}(z)=\mathrm{c}_{1}\left(z-\mathrm{c}_{\mathrm{r}}\right)+\mathrm{c}_{2}\left(n_{c}<1\right)= \begin{cases}\mathrm{c}_{1}\left(z-\mathrm{c}_{\mathrm{r}}\right)+\mathrm{c}_{2}, & n_{c}<1 \\
\mathrm{c}_{1}\left(z-\mathrm{c}_{\mathrm{r}}\right), & n_{c} \geq 1\end{cases} \\
f_{o}(z)=\mathrm{d}_{1} /\left[\left(z / \mathrm{a}_{\mathrm{dis}}^{k^{\prime}}\right)^{\mathrm{d}_{2}^{\mathrm{d}^{\prime}}}\right]
\end{gathered}
$$

where $z$ represents the distance between two points. $n_{c}$ and 1 are the current hunting step number and the number beginning to move to the effective hunting circle(centered as the target, $c_{r}$ as the radius of the circle). $c_{1} 、 d_{1}$, and $d_{2}^{k^{\prime}}$ are designed to optimize the moving path of each robot. $c_{\mathrm{r}}, c_{2}$, and $\mathrm{a}_{\mathrm{dis}}^{k}$ are the effective hunting radius, the approach parameters of chasing, and the distance beginning to strengthen the collision or the obstacle avoidance, respectively. $k^{\prime}=1,2,3$ represents the specific parameters when the objects are robots, static or dynamic obstacles.

For the obstacles that are easy to avoid, i.e., the conditions of following barriers are not satisfied, the mapping function is given as following:

$$
\phi(\sigma)=\left\{\begin{array}{l}
0.75+(\sigma-0.5)^{2}, \quad 0 \leq \sigma \leq 1 \\
-0.75-(\sigma+0.5)^{2}, \quad-1 \leq \sigma<0
\end{array}\right.
$$

where $\sigma$ is the real value of $\cos (\cdot)$ function. The robots can avoid obstacles in advance through the mapping function producing bigger repulsion. Thus it effectively achieves the bionic characteristics of obstacle avoidance.

Assume the direction angles of directed lines $l_{i}$ and $l_{j}$ are $\gamma_{i}$ and $\gamma_{j}$, respectively. In order to determine the angle between the two directed lines, $\gamma_{i j}$ from $l_{i}$ to $l_{j}$ can be computed according to the following formula [32] :

$$
\gamma_{i j}=\operatorname{dagl}\left(\gamma_{i}-\gamma_{j}\right)
$$

where $\operatorname{dagl}(\cdot)$ is given as following:

$$
\operatorname{dagl}(x)= \begin{cases}x-2(n-1) \pi \operatorname{sign}(x), & 2(n-1) \pi \leq|x| \leq 2 n \pi-\pi \\ x-2 n \pi \operatorname{sign}(x), & 2 n \pi-\pi<|x|<2 n \pi\end{cases}
$$

where $\operatorname{sign}(\cdot)$ is sign function. $n$ is an integer. 


\subsection{Hunting Task Model}

Wolves' hunting behavior has inspired the hunting research by the multi-robot systems [17, 33] or swarm robotic systems [30]. This paper focuses on hunting behavior after wolves finding the target or the prey, which mainly includes four stages: target lock, confrontation, chasing, and hunting success. The task mathematical model under an unknown complex environment is as follows.

In the hunting environment, assume there is a set $K \in\{T, S, U, H\}$ that includes the prey (the target) $T=\left\{t_{j}: j=1\right\}$, static obstacles $S=\left\{s_{j}: j=1,2, \cdots, \alpha\right\}$, dynamic obstacles $U=\left\{u_{j}: j=1,2, \cdots, \beta\right\}$, and predators (robots for hunting) $H=\left\{h_{j}: j=1,2, \cdots, m\right\}$. The coordinates of $t_{j}, s_{j}, u_{j}$, and $h_{j}$ in the 2 dimension global coordinate system are $\left(x_{t_{j}}, y_{t_{j}}\right),\left(x_{s_{j}}, y_{s_{j}}\right),\left(x_{u_{j}}, y_{u_{j}}\right)$, and $\left(x_{h_{j}}, y_{h_{j}}\right)$, respectively. $a_{r}^{s}$ and $a_{r}^{U}$ are the distances for the target beginning to strengthen avoiding the static and dynamic obstacles, respectively. $\mathrm{p}_{\mathrm{r}}^{T}, \mathrm{c}_{\mathrm{r}}, \mathrm{s}_{\mathrm{r}}^{T}$, and $\mathrm{s}_{\mathrm{r}}^{H}$ are the radius of the potential domain of the prey, the effective hunting radius, the sensing radius of the target and robots, respectively. Generally, $\mathrm{a}_{\mathrm{r}}^{S} \leq \mathrm{a}_{\mathrm{r}}^{U}<\mathrm{p}_{\mathrm{r}}^{T}<\mathrm{c}_{\mathrm{r}}<\mathrm{s}_{\mathrm{r}}^{T}<\mathrm{s}_{\mathrm{r}}^{H}$. The potential domain of the target $t_{1}$ is $G_{t_{1}}=\left\{(x, y):\left\|(x, y)-\left(x_{t_{1}}, y_{t_{1}}\right)\right\| \leq \mathrm{p}_{\mathrm{r}}^{T}\right\}$. The set of all the robots in the potential domain of the target $t_{1}$ is $N_{T H}=\left\{j \in H: h_{j} \in G_{t_{1}}\right\}=\left\{j \in H:\left\|h_{j}-t_{1}\right\| \leq \mathrm{p}_{\mathrm{r}}^{T}\right\}$. The static obstacles that the target will avoid are $N_{T S}=\left\{j \in S:\left\|s_{j}-t_{1}\right\| \leq \mathbf{a}_{r}^{S}\right\}$. The dynamic obstacles that the target will avoid are $N_{T U}=\left\{j \in U:\left\|u_{j}-t_{1}\right\| \leq \mathrm{a}_{\mathrm{r}}^{U}\right\}$.

In order to describe the confrontation between the individuals and the prey and make the prey effectively avoiding obstacles, we use the concept of "potential" to describe the ability of the robots and obstacles. Assume the potential set in hunting environment is $P=\left\{P_{T}, P_{S}, P_{U}, P_{H}\right\}$, where $P_{T}=\left\{\rho_{t_{i}}\right\}$ is the potential of the target; $P_{S}=\left\{\rho_{s_{j}}\right\}$ is the potential of the static obstacles; $P_{U}=\left\{\rho_{u j}\right\}$ is the potential of the dynamic obstacles; $P_{H}=\left\{\rho_{h_{h}}\right\}$ is the individual potential of swarm robots. The potential that $t_{1}$ can sense is $\tilde{\rho}_{t_{1}}=\left\{\rho_{K}^{e x}, \rho_{K}^{i m}: e x \in N_{T K}, i m \notin N_{T K}, e x \cup i m \in K, N_{T K} \in\left\{N_{T H}\right.\right.$, $\left.N_{T S}, N_{T U}\right\}$, then

$$
\begin{aligned}
& \max \left(\rho_{h_{j}}\right)<\rho_{t_{1}}<\sum \rho_{h_{j}}, \rho_{t_{1}}<\rho_{u_{j}} \leq \rho_{s_{j}}, \rho_{t_{1}}^{*}=\sum_{e \in \in N_{T K}} \rho_{K}^{e x},
\end{aligned}
$$

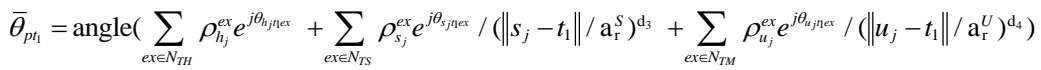

where $\rho_{K}^{e x}$ is called an obvious potential, which is in the potential domain of $t_{1}$ and can be sensed by $t_{1} . \rho_{K}^{\text {in }}$ is called an implicit potential, which is out of the potential domain of $K$ and can be sensed or not. $\rho_{t_{1}}^{*}$ is the sum of obvious potential in the potential domain of $t_{1} \cdot \bar{\theta}_{p_{t}}$ is called the potential angle, and its positive direction is called the escaping direction, which represents the least potential direction of predators, static, and dynamic obstacles sensed by the prey; conversely, its reverse direction is called confrontation direction, which represents

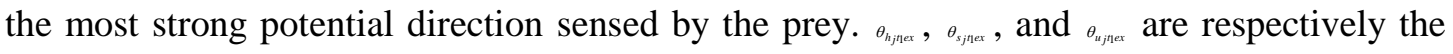
direction angles of hunting robots, static, and dynamic obstacles relative to the prey. $d_{3}$ and $d_{4}$ are designed to optimize $\bar{\theta}_{p_{1}}$. angle(.) is a function for obtaining angle and can be found in MATLAB.

From the above description and (6), motion equations of the dynamic target (prey) in an environment with obstacles are given as follows:

$$
\left\{\begin{array}{l}
\dot{v}_{t_{1}}=\left(\mathrm{v}_{\omega}^{T}-v_{t_{1}}+v_{t_{1}} \times\left(\rho_{t_{1}}^{*}>\rho_{t_{1}}\right)\right) \times\left(\mathrm{v}_{\mathrm{m}}^{T}>v_{t_{t}}\right) \\
\theta_{t_{1}}=\theta_{\omega}^{T}+\left(\rho_{t_{1}}^{*} \neq 0\right) \cdot\left(-\theta_{\omega}^{T}+\bar{\theta}_{\rho_{t}}-\pi+\pi \cdot\left(\rho_{t_{1}}^{*}>\rho_{t_{1}}\right)\right)
\end{array}\right.
$$


where $v_{\omega}^{T}$ and $v_{\mathrm{m}}^{T}$ are respectively the walking speed and maximum speed of the target. Generally there is $\mathrm{v}_{\omega}^{T}<\mathrm{v}_{\mathrm{m}}^{H} \leq \mathrm{v}_{\mathrm{m}}^{T}$, where $\mathrm{v}_{\mathrm{m}}^{H}$ is the maximum speed of the predator. $\left(\mathrm{v}_{\mathrm{m}}^{T}>v_{\mathrm{t}_{\mathrm{t}}}\right)$ is a speed constraint condition. It is 1 if it is true and the prey accelerates, otherwise it is 0 and the prey do not accelerate. $\theta_{\omega}^{T}$ is the initial walking direction angle of the target. When $\left(\rho_{t_{1}^{*}}^{*} \neq 0\right)$, i.e. , the prey finds the hunting robots in its potential domain and it will choose to escape or confront. And if $\left(\rho_{i}^{*}>\rho_{n}\right)$, the prey will choose to escape, which direction is the positive direction of the potential angle, otherwise to confront, which direction is the reverse direction of the potential angle.

The difference of hunting parameters between this paper and [30] is the relative parameters in the environment with unknown cluttered obstacles are given in this paper. Furthermore, $\mathrm{p}_{\mathrm{r}}^{T}$ is not equal to $s_{\mathrm{r}}^{T}$, and that is more realistic. The hunting process is different from [30].

\section{Hunting Algorithm}

In this paper, from the perspective of physics, an autonomous motion model based on simplified virtual-force (called "SVF-model" in short, proposed by the authors) is formed. This model will make the whole swarm implement self-organizing hunting in an unknown complex environment.

\subsection{SVF-Model}

In an unknown complex environment, assume $h_{j}$ can obtain position information of itself, the target $t_{1}$ and two nearest neighbors $a_{j}, b_{j}$ (maybe static convex or non-convex, and dynamic deforming obstacles, robots) in the global-coordinate system $x O y$, shown in Figure 2 . In the relative coordinate system $x^{\prime} O^{\prime} y^{\prime}$, which origin is $h_{j}$, location vectors $\boldsymbol{p}_{j_{1}}, \boldsymbol{p}_{a j}, \boldsymbol{p}_{b j}$, $\boldsymbol{p}_{a b}$ are respectively defined as $\boldsymbol{p}_{j_{1}}=t_{1}-h_{j}, \boldsymbol{p}_{a j}=h_{j}-a_{j}, \boldsymbol{p}_{b j}=h_{j}-b_{j}, \boldsymbol{p}_{a b}=b_{j}-a_{j} . f_{t_{i j}}, f_{a_{j}}$, and $f_{b_{j}}$ are respectively the attraction or repulsion sensed by $h_{j}$ from the target $t_{1}$ and two nearest neighbors $a_{j}, b_{j}$, respectively. The direction of $p_{j_{1}}$ is the positive axis of $y^{\prime}$-axis and the angle from the positive axis of $y^{\prime}$-axis to the positive axis of $x$-axis is defined as $\gamma_{y^{\prime}}$. The direction angle of the positive axis of $x^{\prime}$-axis is $\gamma_{x^{\prime}}=\gamma_{y^{\prime}}-\pi / 2 \cdot \gamma_{f_{i j} j}$ can be determined by:

$$
\gamma_{f_{i, j}}= \begin{cases}\gamma_{y^{\prime}} \pm \pi, & f_{t, j}<0 \\ \gamma_{y^{\prime}}, & f_{t, j} \geq 0\end{cases}
$$

The repulsion angles of two objects $\gamma_{f_{a j}}$ and $\gamma_{f_{j j}}$ are respectively the directed angles from $f_{a_{j}}, f_{b_{j}}$, i.e., vectors $\boldsymbol{p}_{a j}, \boldsymbol{p}_{b j}$, to the positive axis of $x$-axis. $\gamma_{f_{a j} x^{x}}$ and $\gamma_{f_{b j} x^{x}}$ are the repulsion deflection angles of two nearest objects, computed by $\gamma_{f_{f_{j} x^{\prime}}}=\operatorname{dagl}\left(\gamma_{f_{a_{j}}}-\gamma_{x^{\prime}}\right), \gamma_{f_{b j} x^{\prime}}=\operatorname{dagl}\left(\gamma_{f_{b_{j}}}-\gamma_{x^{\prime}}\right) \cdot f_{a b_{j}}$ is the repulsion at $x$ ' axis felt by $h_{j}$, and it is defined as follows:

$$
f_{a b_{j}}=f_{a_{j}}\left(\left\|\boldsymbol{p}_{a j}\right\|\right) \cdot \phi\left(\cos \left(\gamma_{f_{f_{j} x^{\prime}}}\right)\right)+f_{b_{j}}\left(\left\|\boldsymbol{p}_{b_{j}}\right\|\right) \cdot \phi\left(\cos \left(\gamma_{f_{b_{j} x^{\prime}}}\right)\right)
$$

where $f_{a_{j}}\left(\left\|\boldsymbol{p}_{a j}\right\|\right)$ and $f_{b_{j}}\left(\left\|\boldsymbol{p}_{b_{j}}\right\|\right)$ are respectively applying force functions of both objects $a_{j}, b_{j}$ computed by (3). $\gamma_{f_{t a j}}$ can be determined by:

$$
\gamma_{f_{a b j}}= \begin{cases}\gamma_{x^{\prime}} \pm \pi, & f_{a b_{j}}<0 \\ \gamma_{x^{\prime}}, & f_{a b_{j}} \geq 0\end{cases}
$$

$f_{x^{\prime} y^{\prime} j^{\prime}}$ is the whole force of $h_{j}$ constituted by $f_{t i j}$ that is the component at the $y^{\prime}$ axis and $f_{a b_{j}}$ that is the component at the $x^{\prime}$ axis. The directed angle from $f_{x^{\prime} y^{\prime} j}$ to the positive axis of the $x$ axis is the direction angle $\gamma_{f^{\prime} y^{\prime} j}$ of $f_{x^{\prime} y^{\prime} j}$. 
From the above description, we can obtain the required velocity of $h_{j}$ when the target is static:

$$
\boldsymbol{v}_{x^{\prime} y^{\prime} j}=\boldsymbol{f}_{x^{\prime} y^{\prime} j}=\boldsymbol{f}_{t, j}+\boldsymbol{f}_{a b_{j}}=\left|f_{t, j}\left(\left\|\boldsymbol{p}_{j t^{\prime}}\right\|\right)\right| e^{j \gamma_{f h_{j} j}}+\left|f_{a b_{j}}\right| e^{j \gamma_{\gamma a b j}}
$$

where $f_{t j}\left(\left\|\boldsymbol{p}_{i_{1}}\right\|\right)$ is the applying force function of the target $t_{1}$ computed by using (2). Meanwhile, $f_{a b_{j}}, f_{t i j}$ can be directly equivalent to $v_{x^{\prime} j}, v_{y^{\prime} j}$ respectively, which are the velocities of $h_{j}$ at the directions of the $x^{\prime}$ axis and the $y^{\prime}$ axis, respectively. Then there are relationships $v_{x^{\prime} j}=f_{a b_{j}}, v_{y^{\prime} j}=f_{t t^{\prime}}, v_{x^{\prime} y^{\prime} j}=v_{x^{\prime} j}+v_{y^{\prime} j}$, as shown in Figure 2.

\subsection{Individual Control Inputs Design based on SVF-Model}

Assume that robot $h_{j}$ 's expectation moving direction $\theta_{j e}$, the required time $t_{n j}$ for $h_{j}$
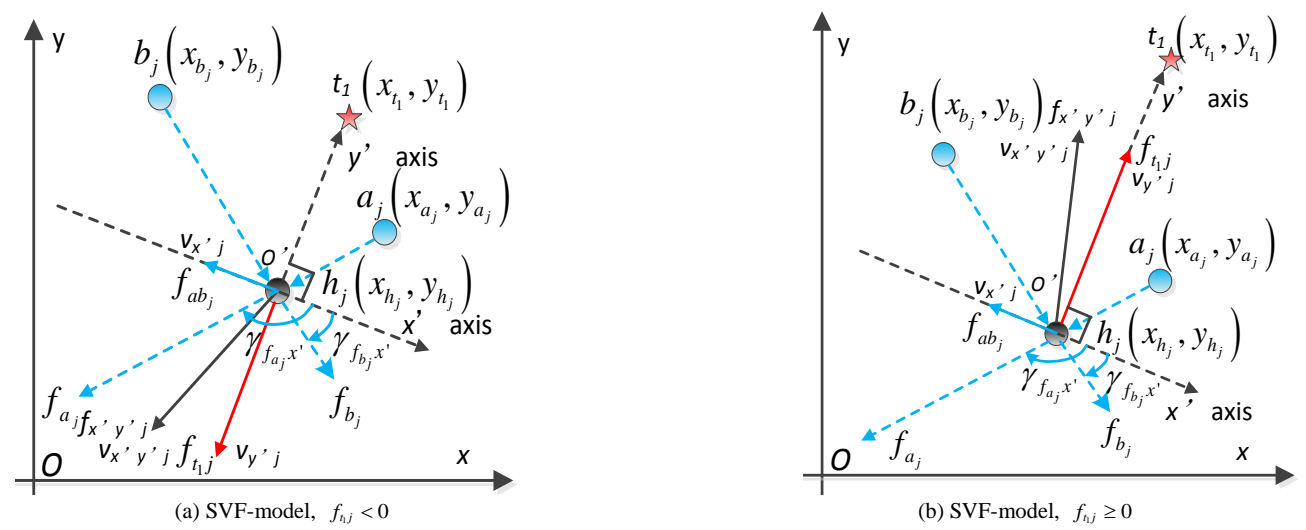

Figure 2. Simplified Virtual-force Model

turning to expectation movement direction $\theta_{j e}$, period of motion $\Gamma$, the actual movement maximum velocity $v_{j f}$, the movement control inputs of $h_{j}$ in an unknown complex environment, i.e., the inputs of (1), are as follows:

When $\Gamma \leq t_{n i}$, the robots only have rotation motions,

$$
\begin{cases}\dot{\omega}_{j}(t)=\omega_{\mathrm{am}}^{H}\left(\omega_{\mathrm{m}}^{H}>\omega_{j}(t)\right), & k \Gamma<t \leq(k+1) \Gamma \\ v_{j}(t)=0, & k \Gamma<t \leq(k+1) \Gamma\end{cases}
$$

When $\Gamma>t_{n j}$, the robots can have rotation and translation motions,

$$
\begin{cases}\dot{\omega}_{j}(t)=\omega_{\mathrm{am}}^{H}\left(\omega_{\mathrm{m}}^{H}>\omega_{j}(t)\right), & k \Gamma<t \leq k \Gamma+t_{n j} \\ v_{j}(t)=0, & k \Gamma<t \leq k \Gamma+t_{n j} \\ \dot{v}_{j}(t)=\mathrm{a}_{\mathrm{m}}^{H}\left(\left|v_{i j}\right|>\left|v_{j}(t)\right|\right), & k \Gamma+t_{n j}<t \leq(k+1) \Gamma \\ \omega_{j}(t)=0, & k \Gamma+t_{n j}<t \leq(k+1) \Gamma\end{cases}
$$

Assume that, in SVF-model $\left\|\boldsymbol{p}_{a j}\right\| \leq\left\|\boldsymbol{p}_{b j}\right\|, \mathrm{f}_{\text {dis }}$ denotes the distance between $h_{j}$ and $a_{j}$ when $h_{j}$ is following of barriers, which can be robots, dynamic deforming obstacles defined as nonconvex obstacles with rotational and translational movement, static convex or non-convex obstacles. The whole expectation velocity $v_{j e}$ and rotation $\theta_{j e}$ are determined by the flow chart of robots following obstacles algorithms (as shown in Figure 3). Thus the swarm robots can avoid the nearest object. 
In Figure 3, the conditions of following barriers are $\left\|\boldsymbol{p}_{a j}\right\| \leq\left\|\boldsymbol{p}_{b j}\right\|<\mathrm{f}_{\text {dis }}$ and $\left\|\boldsymbol{p}_{a b}\right\|<2 \mathrm{f}_{\text {dis }}$. The end conditions of following barriers are $\cos \left(\operatorname{dagl}\left(\gamma_{f t i}-\gamma_{y^{\prime}}\right)\right)>0$ appearing 3 times (i.e., count $\left.>2\right)$ or $\left\|\boldsymbol{p}_{j_{t}}\right\|-\mathrm{c}_{\mathrm{r}}<-1$ or $\left\|\boldsymbol{p}_{a j}\right\|>\mathrm{f}_{\text {dis }}$ and $\left\|\boldsymbol{p}_{a b}\right\|>2 \mathrm{f}_{\text {dis }}$.

If the robots are not in the process of following barriers and do not satisfy the conditions of following barriers or have already ended following barriers, then $v_{j e}, \theta_{j e}$ can be determined by:

$$
v_{j e}=v_{t_{1}}^{\prime}+v_{x^{\prime} y^{\prime} j}, \theta_{j e}=\operatorname{angle}\left(v_{j e}\right)
$$

where $v_{t_{1}}^{\prime}$ is the perceptual velocity of robots to the target. $v_{j e}$ is the expectation velocity vector of robot $h_{j}$ during hunting.

Whether the robots are in the following barriers state or not, $t_{n j}$ and $v_{j f}$ are computed according to the following equations:

$$
\begin{aligned}
& \left\{\begin{array}{l}
t_{n t j 1}=\omega_{\mathrm{m}}^{H} / \omega_{\mathrm{am}}^{H} \\
t_{n j \mathrm{j} 2}=\left[\left[\theta_{j e}-\theta_{j b e f} \mid-\omega_{\mathrm{am}}^{H} \cdot\left(t_{n t 1}\right)^{2} / 2\right] / \omega_{\mathrm{m}}^{H}\right. \\
t_{n j i}=\left(t_{n j i}+t_{n j i 2}\right)\left(\left|\theta_{j e}-\theta_{j b e f}\right| \geq \omega_{\mathrm{am}}^{H} \cdot\left(t_{n j i}\right)^{2} / 2\right)+\sqrt{2\left|\theta_{j e}-\theta_{j b e f}\right| / \omega_{\mathrm{am}}^{H}} \times\left(\left|\theta_{j e}-\theta_{j b e f}\right|<\omega_{\mathrm{am}}^{H} \cdot\left(t_{n j i 1}\right)^{2} / 2\right)
\end{array}\right. \\
& \left\{\begin{array}{l}
\Gamma_{m t j}=\Gamma-t_{n i j} \\
v_{j c}=\left(\Gamma_{t m j}-\sqrt{\Gamma_{m i j}{ }^{2}-2 v_{j e} \Gamma / \mathrm{a}_{\mathrm{m}}^{H}}\right) \mathrm{a}_{\mathrm{m}}^{H} \times\left(\Gamma_{t m j}{ }^{2} \geq 2 v_{j} \Gamma / \mathrm{a}_{\mathrm{m}}^{H}\right)+\mathrm{v}_{\mathrm{m}}^{H}\left(\Gamma_{m t}{ }^{2}<2 v_{j e} \Gamma / \mathrm{a}_{\mathrm{m}}^{H}\right) \\
v_{j f}=v_{j c}\left(v_{j c} \leq \mathrm{v}_{\mathrm{m}}^{H}\right)+\mathrm{v}_{\mathrm{m}}^{H}\left(v_{j c}>\mathrm{v}_{\mathrm{m}}^{H}\right)
\end{array}\right.
\end{aligned}
$$

In the above equations, the functions without containing time all denote the calculation at $k \Gamma$ and keep the same on during $[k \Gamma,(k+1) \Gamma)$, where $\theta_{j e}$ and $\theta_{j b f}$ are respectively the movement direction of the next step and the movement direction of the previous step. $t_{n j}$ is the required time for turning according to the maximum angle acceleration and the maximum angle velocity. $t_{n j i}$ is the required time for accelerating to maximum angle velocity according to the maximum angle acceleration. $t_{n j 2}$ is the required time from the moment of reaching the maximum angle velocity to turning to the expectation movement direction $\theta_{j e} . v_{j c}$ is the compensation speed according to expectation speed.

\subsection{Hunting Algorithm Steps}

According to the constructing hunting environment in section 2.2 and the movement mathematical equation constructed by (7), the pseudo codes for the hunting algorithm are shown in Figure 4. 


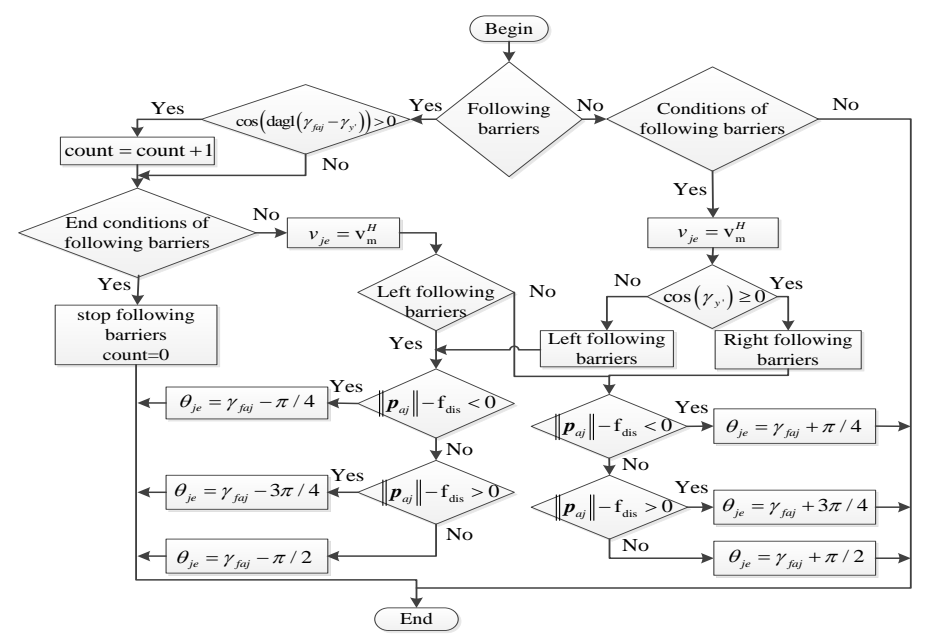

Figure 3. The Flow Chart of Robots Following Obstacles Algorithms

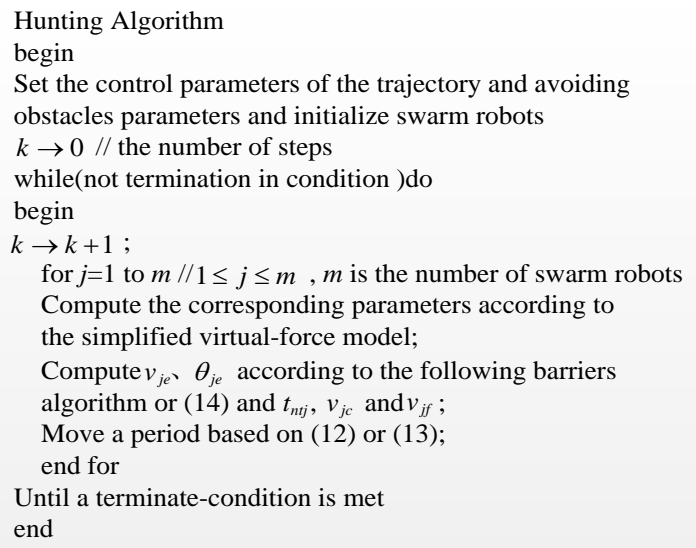

Figure 4. Pseudocode for the Hunting Algorithm

where the terminate-condition are all individuals satisfying $\left\|\boldsymbol{p}_{j_{t}}\right\|-\mathrm{c}_{\mathrm{r}} \mid<\varepsilon_{1}$ and $\left\|\boldsymbol{p}_{a j}\right\|-\left\|\boldsymbol{p}_{b_{j}}\right\|<\varepsilon_{2}$.

\section{Stability Analysis}

We follow the stability analysis method used in [30]. In order to find the convergence conditions of the hunting algorithm when the conditions of the following barriers are not meet, system deviation is decomposed into target distance deviation $\delta_{j y^{\prime}}=\left\|\mathbf{p}_{j_{t_{t}}}\right\|-\mathrm{c}_{\mathrm{r}}$ [30] and two nearest-neighboring robots distance deviation $\delta_{j a b x^{\prime}}=\left(s_{f a j x^{\prime}}\left\|\boldsymbol{p}_{a j}\right\|+s_{f j j x^{\prime}}\left\|\boldsymbol{p}_{b j}\right\|+d_{j o}\right) / 2$, where $s_{f a j x}=\operatorname{sign}(-$

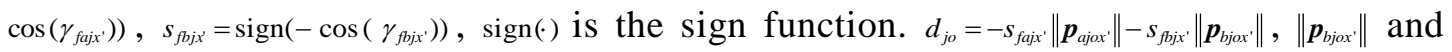
$\left\|\boldsymbol{p}_{a j x^{x}}\right\|$ are the distances from the force balance point $h_{j o}$ to $b_{j}$ and $a_{j}$ that are the left and right two nearest neighbors. $\left\|\boldsymbol{p}_{b j o x}\right\|$ and $\left\|\boldsymbol{p}_{a j o x}\right\|$ meet the relationship between angles and sides in a triangle on the effective hunting circle, i.e., the following (17), and has a unique solution: 


$$
\begin{aligned}
& \|\| \boldsymbol{p}_{b j o x} \|=2 c_{\mathrm{r}} \cos \left(\gamma_{f j j x^{\prime}}+\pi-\pi / 2\right) \\
& \left\|\boldsymbol{p}_{\text {ajox }}\right\|=2 \mathrm{c}_{\mathrm{r}} \cos \left(\pi / 2-\left(\gamma_{\text {fajox }}+\pi\right)\right) \\
& \left\{\left\|\boldsymbol{p}_{a b}\right\|=2 \mathrm{c}_{\mathrm{r}} \sin \left[(1 / 2)\left[\pi-2\left(\gamma_{f j j o x^{\prime}}+\pi-\pi / 2\right)+\pi-2\left(\pi / 2-\left(\gamma_{\text {fajox }}+\pi\right)\right)\right]\right]\right.
\end{aligned}
$$

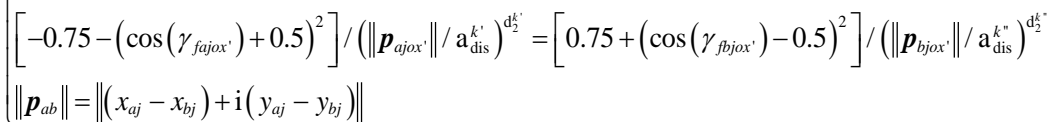

where $\gamma_{f j j x^{\prime}}$ and $\gamma_{f a j o x}$ are respectively the direction angles from the repulsion of $b_{j}$ and $a_{j}$ sensed by $h_{j o}$ to the positive axis of the $x^{\prime}$ axis origined at $h_{j o}$. The range of $\gamma_{f f j o x}$ and $\gamma_{f a j o x^{\prime}}$ are $(-\pi / 2,0)$ and $(-\pi,-\pi / 2)$, respectively. $k^{\prime}=1,2,3 ; k^{\prime \prime}=1,2,3$. When $\delta_{j y^{\prime}}=0, \delta_{j a b x^{\prime}}=0(j=1,2, \ldots, m)$, the ideal hunting formation is formed. Therefore, to obtain the stability conditions of the selforganizing hunting systems, there is only need to infer the conditions that the systems should be satisfied when $\delta_{j y^{\prime}} \rightarrow 0, \delta_{j a b x^{\prime}} \rightarrow 0(j=1,2, \ldots, m)$.

Consider the discretized positional deviation as:

$$
\begin{aligned}
& \delta_{j y^{\prime}}(k)=\left\|\boldsymbol{p}_{j t_{1}}(k)\right\|-c_{\mathrm{r}}, j=1,2, \ldots m, \\
& \delta_{j a b x^{\prime}}(k)=\left(s_{f a j j^{\prime}}(k)\left\|\boldsymbol{p}_{a j}(k)\right\|+s_{f j j^{\prime}}(k)\left\|\boldsymbol{p}_{b j}(k)\right\|+d_{j o}(k)\right) / 2, j=1,2, \ldots m .
\end{aligned}
$$

where $s_{f a j x^{\prime}}(k)=\operatorname{sign}\left(-\cos \left(\gamma_{f j x^{\prime}}(k)\right)\right), s_{f j j^{\prime}}(k)=\operatorname{sign}\left(-\cos \left(\gamma_{f b j x^{\prime}}(k)\right)\right), d_{j o}(k)=-s_{f a j x^{\prime}}(k)\left\|\boldsymbol{p}_{\text {ajox }}(k)\right\|-s_{f f j x^{\prime}}(k)\left\|\boldsymbol{p}_{\text {bjox }}(k)\right\|$.

Let dynamic disturbance $v_{t} \equiv 0$, based on SVF-model, without considering the physical constraints of the robots themselves (such as angular velocity and linear velocity). They move according to the desired velocity and direction at each step. The discretized forms $v_{y^{\prime} j}(k)$ and $v_{x^{\prime} j}(k)$ of $v_{y^{\prime} j}$ and $v_{x^{\prime} j}$ are expressed respectively as follows:

$$
\begin{gathered}
v_{y^{\prime} j}(k)=f_{t \mid j}(k)=c_{1}\left(\left\|p_{j t_{1}}(k)\right\|-\mathrm{cr}_{\mathbf{r}}\right)+\mathrm{c}_{2}\left(n_{c}<1\right) \\
v_{x^{\prime} j}(k)=f_{a b_{j}}(k)=f_{a j}\left(\left\|p_{a j}(k)\right\|\right) \cdot \phi_{a j} x^{\prime} \\
(k)+f_{b_{j}}\left(\left\|p_{b j}(k)\right\|\right) \cdot \phi_{b_{j} x^{\prime}}(k)
\end{gathered}
$$

where $\phi_{a j x^{\prime}}(k)=\phi\left(\cos \left(\gamma_{f a j} x^{\prime}(k)\right)\right), \phi_{b j x^{\prime}}(k)=\phi\left(\cos \left(\gamma_{f b_{j} x^{\prime}}(k)\right)\right)$. Therefore, we can obtain the individual deviation equations of the autonomous motion:

$$
\begin{gathered}
\delta_{j y^{\prime}}(k+1)=\delta_{j y^{\prime}}(k)-v_{y^{\prime} j}(k) \Gamma \\
\delta_{j a b x^{\prime}}(k+1)=\delta_{j a b x^{\prime}}(k)-v_{x^{\prime} j}(k) \Gamma
\end{gathered}
$$

\subsection{Theorem 1}

Theorem 1. In the obstacles environment without satisfying the conditions of following barriers, if all the robots satisfy $n_{c} \geq 1,0<\mathrm{c}_{1} \Gamma<2$, and (20), then the system origin equilibrium state, i.e., $\Delta_{y^{\prime}}(k)=\left(\delta_{1 y^{\prime}}, \delta_{2 y^{\prime}}, \cdots, \delta_{m y^{\prime}}\right)^{\mathrm{T}}=0$, is asymptotic stable in the large field.

Proof. Substituting (18) into (20), we obtain:

$$
\delta_{j y^{\prime}}(k+1)=\delta_{j y^{\prime}}(k)-\left(\mathrm{c}_{1}\left(\left|\mathbf{p}_{j t_{1}}(\mathrm{k})\right|-\mathrm{c}_{\mathrm{r}}\right)+\mathrm{c}_{2}\left(n_{c}<1\right)\right) \Gamma
$$

When $n_{c} \geq 1$, (19) is transformed into:

$$
\delta_{j y^{\prime}}(k+1)=\delta_{j y^{\prime}}(k)-\left(\mathrm{c}_{1}\left(\left\|\mathbf{p}_{j t_{1}}(\mathrm{k})\right\|-\mathrm{c}_{\mathrm{r}}\right)\right) \Gamma=\delta_{j y^{\prime}}(k)-\left(\mathrm{c}_{1} \delta_{j y^{\prime}}(k)\right) \Gamma=\delta_{j y^{\prime}}(k)\left(1-\mathrm{c}_{1} \Gamma\right)
$$

Let Lyapunov function $V_{y^{\prime}}\left(\Delta_{y^{\prime}}(k)\right)=\sum_{j=1}^{m}\left|\delta_{j y^{\prime}}(k)\right|, V_{y^{\prime}}\left(\Delta_{y^{\prime}}(k)\right)$ is positive definite and $V_{y^{\prime}}(0)=0$. Furthermore, we obtain:

$$
\Delta V_{y^{\prime}}\left(\Delta_{y^{\prime}}(k)\right)=V_{y^{\prime}}\left(\Delta_{y^{\prime}}(k+1)\right)-V_{y^{\prime}}\left(\Delta_{y^{\prime}}(k)\right)=\sum_{j=1}^{m}\left|\delta_{j y^{\prime}}(k+1)\right|-\sum_{j=1}^{m}\left|\delta_{j y^{\prime}}(k)\right|=\sum_{j=1}^{m}\left|\delta_{j y^{\prime}}(k)\left(1-c_{1} \Gamma\right)\right|-\sum_{j=1}^{m}\left|\delta_{j y^{\prime}}(k)\right|
$$




$$
\leq-\left(1-\left|\left(1-\mathrm{c}_{1} \Gamma\right)\right|\right) \sum_{j=1}^{m}\left|\delta_{j y^{\prime}}(k)\right|
$$

In order that $\Delta V_{y^{\prime}}\left(\Delta_{y^{\prime}}(k)\right)$ is negative definite, it is sufficient that $0<\mathrm{c}_{1} \Gamma<2$. Additionally, when $\left\|\Delta_{y^{\prime}}(k)\right\| \rightarrow \infty, V_{y^{\prime}}\left(\Delta_{y^{\prime}}(k)\right) \rightarrow \infty$. Therefore, by the Lyapunov stability theorem of a discrete system, we derive that the system origin equilibrium state $\Delta_{y^{\prime}}(k)=0$ is asymptotic stability in the large field and $0<c_{1} \Gamma<2$ is a sufficient condition. Theorem 1 is verified.

From Theorem 1, we know that all the robots in the swarm will finally converge to the circle of radius $c_{r}$ centered at the target. Then if we want to obtain the formation of uniform distribution, we need to consider the convergence of $\delta_{j a b x^{\prime}}(k)$, i.e., (21), when || $\mathbf{p}_{j t_{1}}(k)||-c_{\mathrm{r}} \mid<\varepsilon_{1},(j=1,2, \ldots, m)$. Additionally, the stability analyses results of the system origin equilibrium state $\Delta_{y^{\prime}}(k)=0$ in Theorem 1 can be applied to a no obstacle environment without any modification. The reason for that is whether there are obstacles or not, the attraction or repulsion from the target in SVF-model isn't affected. Therefore, the velocity approaching to the effective hunting circle isn't affected and the stability analyses in Theorem 1 is the same as in the environment without obstacles.

\subsection{Theorem 2}

Theorem 2. In the obstacles environment without satisfying the conditions of following barriers, if all the robots satisfy $0<\Gamma \mathrm{d}_{1} \mu<2$ and (21), then the system origin equilibrium state, i.e., $\Delta_{a b x^{\prime}}(k)=\left(\delta_{1 a b x^{\prime}}, \delta_{2 a b x^{\prime}}, \cdots, \delta_{\text {mabx }}\right)^{\mathrm{T}}=0$, is asymptotic stable in the large field, where $\mu=\max \mu_{j}(k)$,

$$
\begin{aligned}
\mu_{j}(k)= & {\left[\phi\left(\cos \left(\gamma_{f_{a j} x^{\prime}}(k)\right)\right) /\left(\left(\left\|\boldsymbol{p}_{a j}(k)\right\| / \mathrm{a}_{\mathrm{dis}}^{k^{\prime}}\right)^{\mathrm{d}_{2}^{k^{\prime}}}\right)+\phi\left(\cos \left(\gamma_{f_{b j} x^{\prime}}(k)\right)\right) /\left(\left(\left\|\boldsymbol{p}_{b j}(k)\right\| / \mathrm{a}_{\mathrm{dis}}^{k^{\prime \prime}}\right)^{\mathrm{d}_{2}^{k^{*}}}\right)\right] / \delta_{j a b x^{\prime}}(k)>0(j=1, \ldots, m ; n \geq 1,} \\
& \left.k=0,1, \ldots, n ; k^{\prime}=1,2,3 ; k^{\prime \prime}=1,2,3\right) .
\end{aligned}
$$

Proof. Substituting (19) into (21), we obtain:

$$
\begin{aligned}
\delta_{j a b x^{\prime}}(k+1) & =\delta_{j a b x^{\prime}}(k)-f_{a b_{j}}(k) \Gamma=\delta_{j a b x^{\prime}}(k)-\left[f_{a_{j}}\left(\left\|\boldsymbol{p}_{a j}(k)\right\|\right) \cdot \phi\left(\cos \left(\gamma_{f_{a j} x^{\prime}}(k)\right)\right)+f_{b_{j}}\left(\left\|\boldsymbol{p}_{b j}(k)\right\|\right) \cdot \phi\left(\cos \left(\gamma_{f_{b_{j} x^{\prime}}}(k)\right)\right)\right] \Gamma \\
& =\delta_{j a b x^{\prime}}(k)-\left[\phi\left(\cos \left(\gamma_{f_{a j} x^{\prime}}(k)\right)\right) /\left(\left(\left\|\boldsymbol{p}_{a j}(k)\right\| / \mathrm{a}_{\mathrm{dis}}^{k^{\prime}}\right)^{\mathrm{d} k^{\prime}}\right)+\phi\left(\cos \left(\gamma_{f_{b j} x^{\prime}}(k)\right)\right) /\left(\left(\left\|\boldsymbol{p}_{b j}(k)\right\| / \mathrm{a}_{\mathrm{dis}}^{k^{k^{\prime \prime}}}\right)^{\mathrm{d}^{k^{\prime}}}\right)\right] \Gamma \mathrm{d}_{1}
\end{aligned}
$$

Since each of robot only has one kind of equilibrium point when the robots are on the effective hunting circle and $f_{a b_{j}}(k)=0 \quad(j=1,2, \ldots, m)$. Especially, when the two nearest neighbors of every individual are respectively its left and right sides robots, $f_{a b_{j}}(k)$ always eliminates the existence of $\delta_{j a b x^{\prime}}(k)$ such that all the robots reach the balance of force. And therefore the sign of $f_{a b_{j}}(k)$ and $\delta_{j a b x^{\prime}}(k)$ are the same. Since $\mathrm{d}_{1}>0$ and $\Gamma>0$, the sign of $\left[\phi\left(\cos \left(\gamma_{f_{a_{j}} x^{\prime}}(k)\right)\right) /\left(\left(\left\|\boldsymbol{p}_{a j}(k)\right\| / \mathrm{a}_{\mathrm{dis}}^{k^{\prime}}\right)^{\mathrm{d}_{k^{\prime}}}\right)+\phi\left(\cos \left(\gamma_{f_{b j} x^{\prime}}(k)\right)\right) /\left(\left(\left\|\boldsymbol{p}_{b j}(k)\right\| / \mathrm{a}_{\mathrm{dis}}^{k^{\prime \prime}}\right)^{\mathrm{d}_{2}^{k^{\prime \prime}}}\right)\right]$ and $\delta_{j a b x^{\prime}}(k)$ are the same. Thus, the above equation can be written as:

$$
\delta_{\text {jabx' }}(k+1)=\delta_{\text {jabx' }}(k)-\Gamma \mathrm{d}_{1} \mu_{j}(k) \delta_{\text {jabx' }}(k)=\delta_{\text {jabx' }}(k)\left(1-\Gamma \mathrm{d}_{1} \mu_{j}(k)\right)
$$

where, $\mu_{j}(k)$ is computed as follows:

$$
\mu_{j}(k)=\left[\phi\left(\cos \left(\gamma_{f_{a_{j}} x^{\prime}}(k)\right)\right) /\left(\left(\left\|\boldsymbol{p}_{a j}(k)\right\| / \mathrm{a}_{\mathrm{dis}}^{k^{\prime}}\right)^{\mathrm{d}_{2}^{k^{\prime}}}\right)+\phi\left(\cos \left(\gamma_{f_{b_{j}} x^{\prime}}(k)\right)\right) /\left(\left(\left\|\boldsymbol{p}_{b j}(k)\right\| / \mathrm{a}_{\mathrm{dis}}^{k^{\prime \prime}}\right)^{\mathrm{d}_{2}^{k^{\prime \prime}}}\right)\right] / \delta_{j a b x^{\prime}}(k)>0
$$

Construct Lyapunov function $V_{a b x^{\prime}}\left(\Delta_{a b x^{\prime}}(k)\right)=\sum_{j=1}^{m}\left|\delta_{j a b x^{\prime}}(k)\right|$. When $\Delta_{a b x^{\prime}}(k) \neq 0, V_{a b x^{\prime}}\left(\Delta_{a b x^{\prime}}(k)\right)>0$ and $V_{a b x^{\prime}}\left(\Delta_{a b x^{\prime}}(k)\right)$ is positive definite. Furthermore, we obtain:

$$
\Delta V_{a b x^{\prime}}\left(\Delta_{a b x^{\prime}}(k)\right)=V_{a b x^{\prime}}\left(\Delta_{a b x^{\prime}}(k+1)\right)-V_{a b x^{\prime}}\left(\Delta_{a b x^{\prime}}(k)\right)=\sum_{j=1}^{m}\left|\delta_{j a b x^{\prime}}(k+1)\right|-\sum_{j=1}^{m}\left|\delta_{j a b x^{\prime}}(k)\right|
$$




$$
\begin{aligned}
& =\sum_{j=1}^{m}\left|\delta_{j a b x^{\prime}}(k)\left(1-\Gamma \mathrm{d}_{1} \max \mu_{j}(k)\right)\right|-\sum_{j=1}^{m}\left|\delta_{j a b x^{\prime}}(k)\right| \leq \sum_{j=1}^{m}\left|\delta_{j a b x^{\prime}}(k)\right|\left|1-\Gamma \mathrm{d}_{1} \mu\right|-\sum_{j=1}^{m}\left|\delta_{j a b x^{\prime}}(k)\right| \\
& =-\left(1-\left|1-\Gamma \mathrm{d}_{1} \mu\right|\right) \sum_{j=1}^{m}\left|\delta_{j a b x^{\prime}}(k)\right|
\end{aligned}
$$

where assume $\mu$ appears the maximum value at $n_{1}$ step, i.e., $\mu=\max \mu_{j}(k)(j=1,2, \cdots, m ; k=0,1$, $\left.\cdots, n_{1}\right)$. If at $n_{1}$ step, $\mu$ doesn't reach the maximum value, then $\mu$ appears the maximum value at the latest $n_{1}+q$ step. Let $n=n_{1}+q$, there is $\mu=\max \mu_{j}(k)(j=1,2, \cdots, m ; k=0,1, \cdots, n)$. In order that $\Delta V_{a b x^{\prime}}\left(\Delta_{a b x^{\prime}}(k)\right)$ is negative definite, it is sufficient that $0<\Gamma \mathrm{d}_{1} \mu<2$. Additionally, when $\left\|\Delta_{a b x^{\prime}}(k)\right\| \rightarrow \infty, V_{a b x^{\prime}}\left(\Delta_{a b x^{\prime}}(k)\right) \rightarrow \infty$. According to the Lyapunov stability theorem of discrete system, we derive that the system origin equilibrium state $\Delta_{a b x}(k)=0$ is asymptotic stability in the large field and $0<\Gamma \mathrm{d}_{1} \mu<2$ is a sufficient condition for that. Theorem 2 is verified.

When the target is static in the obstacles environment without satisfying the conditions of following barriers, the hunting systems are stable as long as they satisfies Theorem 1 and Theorem 2, i.e., $0<\Gamma<\min \left(2 / \mathrm{c}_{1}, 2 /\left(\mathrm{d}_{1} \mu\right)\right)$. Although the constraints of actual physical systems (such as the velocity and accelerate of robots) may let the convergence of the hunting systems become slow, all robots will converge to an expected force-balance formation on the effective hunting circle at a certain precision in finite time. Usually, the formation is not evenly distributed, but the systems are stable.

Generally speaking, the parameters of avoiding static obstacles and dynamic obstacles $a_{\text {dis }}^{2}, a_{\text {dis }}^{3}, d_{2}^{2}$, and $d_{2}^{3}$ are bigger than the parameters of avoiding robots $a_{\mathrm{dis}}^{1}$ and $\mathrm{d}_{2}^{1}$. The reason for that is it is beneficial for robots keeping away from obstacles, which cannot coordinate with robots, and try to avoid collision damage. Particularly, parameters adjustment method is given here when the systems are unstable, i.e., decreasing $\mathrm{c}_{1}, \mathrm{~d}_{1}$ or $\Gamma$, et al. For the actual hunting experimental physics systems, the parameters can also be changed self-adaptively according to this condition to make the oscillation systems become stable.

\subsection{Theorem 3}

Theorem 3. In the obstacles environment without satisfying the conditions of following barriers and $a_{\text {dis }}^{1}=a_{\text {dis }}^{2}=a_{\text {dis }}^{3}, d_{2}^{1}=d_{2}^{2}=d_{2}^{3}$ or in the no obstacles environment without satisfying the conditions of following barriers, if all the robots satisfy $0<\Gamma \mathrm{d}_{1}\left(\mathrm{a}_{\mathrm{dis}}^{1}\right)^{\mathrm{d}^{\frac{1}{2}}} \mu^{i}<2$ and (21), then the system origin equilibrium state, i.e., $\Delta_{a b x^{\prime}}(k)=\left(\delta_{1 a b x^{\prime}}, \delta_{2 a b x^{\prime}}, \cdots, \delta_{m a b x^{\prime}}\right)^{\mathrm{T}}=0$, is asymptotic stable in the

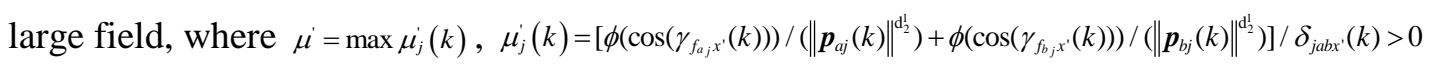
$\left(j=1, \ldots, m ; n^{\prime} \geq 1, k=0,1, \ldots, n^{\prime}\right)$.

Because the derivation process of the Theorem 3 is similar toTheorem 2, it is omitted here. From the Theorem 3, we know that it is the special case of Theorem 2.

The above stability analysis is for a static target. For a dynamic target, there is a sufficient condition for swarm robots to form the hunting formation of force balance as shown below.

\subsection{Theorem 4}

Theorem 4. For a dynamic target, to form the hunting formation of force balance, a lower limit of $\Gamma$ is given: $\Gamma>\max \left(t_{\text {mit }}, t_{\text {malv }}\right)$, where $t_{\text {mit }}$ and $t_{\text {malv }}$ are the minimum of $\Gamma$ satisfying $\Gamma_{m t^{2}}{ }^{2}$ 
$2 \mathrm{v}_{\omega}^{\mathrm{V}} \Gamma / \mathrm{a}_{m}^{H}>0$ and the actual movement velocity of swarm robots for hunting the target with walking speed to escape, respectively. $t_{\text {mit }}$ and $t_{\text {malv }}$ are calculated by $t_{m i t}=\left(t_{n t}+\mathrm{v}_{\omega}^{T} / \mathrm{a}_{\mathrm{m}}^{H}\right)+$ $\sqrt{2 \mathrm{v}_{\omega}^{T} t_{n t} / \mathrm{a}_{\mathrm{m}}^{H}+\left(\mathrm{v}_{\omega}^{T} / \mathrm{a}_{\mathrm{m}}^{H}\right)^{2}}$ and $t_{\text {malv }}=\left(2 t_{n t} \mathrm{v}_{\mathrm{m}}^{H}+\left(\mathrm{v}_{\mathrm{m}}^{H}\right)^{2} / \mathrm{a}_{\mathrm{m}}^{H}\right) /\left(2\left(\mathrm{v}_{\mathrm{m}}^{H}-\mathrm{v}_{\omega}^{T}\right)\right)$, respectively.

Proof. For a dynamic target, to form the hunting formation of force balance, a lower limit of $\Gamma$ is that the movement time of each step is enough to make individuals rotate for $180^{\circ}$ and can reach the velocities in excess of equivalent to the prey's walking speed to move a time step (but do not exceed the maximum speed of the robots $\mathrm{v}_{\mathrm{m}}^{H}$ ), i.e., satisfying the conditions in (15) and (16):

$$
\left\{\begin{array}{l}
t_{n t}=t_{n t 1}+t_{n t 2} \\
t_{n t 1}=\omega_{\mathrm{m}}^{H} / \omega_{\mathrm{am}}^{H} \\
t_{n t 2}=\left[\pi-\omega_{\mathrm{am}}^{H} \cdot t_{n t 1}{ }^{2} / 2\right] / \omega_{\mathrm{m}}^{H} \\
\Gamma_{t n t}=\Gamma-t_{n t} \\
\Gamma_{t n t}{ }^{2}-2 \mathrm{v}_{\omega}^{T} \Gamma / \mathrm{a}_{\mathrm{m}}^{H}>0 \\
\left(\Gamma_{t t t}-\sqrt{\Gamma_{t t n t}{ }^{2}-2 v_{j e} \Gamma / \mathrm{a}_{\mathrm{m}}^{H}}\right) \mathrm{a}_{\mathrm{m}}^{H} \leq \mathrm{v}_{\mathrm{m}}^{H}
\end{array}\right.
$$

Through solving, we can obtain $\Gamma>\max \left(t_{\text {mit }}, t_{\text {malv }}\right)$, where $t_{\text {mit }}=\left(t_{n t}+\mathrm{v}_{\omega}^{T} / \mathrm{a}_{\mathrm{m}}^{H}\right)+\sqrt{2 \mathrm{v}_{\omega}^{T} t_{n t} / \mathrm{a}_{\mathrm{m}}^{H}+\left(\mathrm{v}_{\omega}^{T} / \mathrm{a}_{\mathrm{m}}^{H}\right)^{2}}$, $t_{\text {malv }}=\left(2 t_{n t} \mathrm{v}_{\mathrm{m}}^{H}+\left(\mathrm{v}_{\mathrm{m}}^{H}\right)^{2} / \mathrm{a}_{\mathrm{m}}^{H}\right) /\left(2\left(\mathrm{v}_{\mathrm{m}}^{H}-\mathrm{v}_{\mathrm{\omega}}^{T}\right)\right)$. Theorem 4 is verified.

Considering the above Theorem 1, Theorem 2, and Theorem 4, when the dynamic target is escaping at walking speed, a sufficient condition for swarm robots hunting success is as follows:

$$
\max \left(t_{\text {mit }}, t_{\text {malv }}\right)<\Gamma<\min \left(2 / \mathrm{c}_{1}, 2 /\left(\mathrm{d}_{1} \mu\right)\right)
$$

Considering the above Theorem 1, Theorem 3, and Theorem 4, we can obtain a sufficient condition for swarm robots hunting success for another special case, when the dynamic target is escaping at walking speed, is as follows:

$$
\max \left(t_{\text {mit }}, t_{\text {manu }}\right)<\Gamma<\min \left(2 / \mathrm{c}_{1}, 2 /\left(\mathrm{d}_{1}\left(\mathrm{a}_{\text {dis }}^{1}\right)^{\mathrm{d}_{2}} \mu\right)\right)
$$

Since the target doesn't rotate $180^{\circ}$ at each step when it is escaping, robots do not need to rotate $180^{\circ}$ at each step neither. Therefore, for most instances the swarm robots can also hunt successfully when $\Gamma$ is less than the lower limit given by (26) or (27).

\subsection{Special Case 1}

For the static non-convex and convex obstacles environment with satisfying the conditions of following barriers, the following barriers robots can avoid non-convex and convex obstacles safely as long as the following barriers robots can keep the latest positional information of the target (by the sense of themselves or communicating with others to get the latest positional information of the target), then as long as time step length $\Gamma$ of the following barriers robots satisfies (26) or (27), and the hunting systems are stable.

If the following barriers robots lost the sense to the target and the communication with others and lost, and if the number of lost robots is less, since the number of swarm robots is more, it doesn't affect the stability of the hunting systems. This also reflects good robustness of the swarm robotic systems.

\subsection{Special Case 2}

For dynamic deforming obstacles environment with satisfying the conditions of following barriers, the robots need to avoid deforming obstacles. It is necessary to analyze the movements' characteristics between robots and deforming obstacles. There are two most extreme cases of movement between robots and deforming obstacles: one is that both of them 
move with the same direction, and the other one is that both of them move with the opposite direction.

The most demanding situations for robots in the first case are that robots and obstacles are very close, and the robots need to rotate $180^{\circ}$, then the robots depart the obstacles at the angle of $45^{\circ}$ with the maximum velocity. And the obstacles move with the maximum speed at the same direction with the robots. In this situation to make obstacle avoidance, we can determine the constraint for the maximum velocity of the deforming obstacles.

The most demanding situations for robots in the second case are that the distance between robots and deforming obstacles approximate $\mathrm{f}_{\text {dis }}$, and the robots do not need to rotate but rapidly accelerate to maximum speed to move oppositely with the deforming obstacles. And the deforming obstacles move with the maximum speed. In this situation to make obstacle avoidance, we can determine another constraint for the maximum velocity of the deforming obstacles.

Therefore, as long as the deforming obstacles satisfy the maximum speed allowed in the above two cases (take smaller value in two cases as the maximum speed of deforming obstacles), and as long as the linear velocity of any part of the obstacles is not greater than this value, but regardless of what kind of sports of the deforming obstacles, due to the robots have the velocity components at the perpendicular direction to the line from the robots to $a_{j}$ in the deforming obstacles and always keep the target location updating, the robots all can follow barriers successfully and join in the hunting formation finally. Thus, the whole hunting systems are stable.

To determine the maximum velocity of the deforming obstacles $v_{\mathrm{m}}^{U}$, according to the value of $\Gamma$, there are three situations to discuss. Define $t_{n 1}=\omega_{\mathrm{m}}^{H} / \omega_{\mathrm{am}}^{H}, t_{n t 2}=\left[\pi-\omega_{\mathrm{am}}^{H} \cdot t_{n 1}{ }^{2} / 2\right] / \omega_{\mathrm{m}}^{H}$, $t_{n t}=t_{n t 1}+t_{t r 2}, \Gamma_{t t t}=\Gamma-t_{n t}$. When (1) $\Gamma_{t t t} \leq 0$, since there is no time for following robots to translate except for rotating, $\mathrm{v}_{\mathrm{m}}^{U}=0$. When (2) $0<\Gamma_{m t} \leq \mathrm{v}_{\mathrm{m}}^{H} / \mathrm{a}_{\mathrm{m}}^{H}, \mathrm{v}_{\mathrm{m}}^{U}$ can be determined by $\mathrm{v}_{\mathrm{m}}^{U}=\min \left(v_{h o l}, v_{\text {opl }}\right)$, where $v_{h o l}$ and $v_{o p l}$ represent, when $0<\Gamma_{t o t} \leq \mathrm{v}_{\mathrm{m}}^{H} / \mathrm{a}_{\mathrm{m}}^{H}$, the allowed maximum velocity of the dynamic deforming obstacles without collision with robots when they move at same direction and at opposite direction, respectively. $v_{h o l}$ and $v_{o p l}$ can be calculated by $v_{h o l}=\mathrm{a}_{\mathrm{m}}^{H} \Gamma_{m t}^{2} \cos (\pi / 4) /(2 \Gamma)$ and $v_{\text {opl }}=\left(\left(\mathrm{f}_{\text {dis }}-\mathrm{a}_{\mathrm{m}}^{H} \Gamma^{2} / 2\right) / \Gamma\right)\left(\Gamma \leq \mathrm{v}_{\mathrm{m}}^{H} / \mathrm{a}_{\mathrm{m}}^{H}\right)+\left(\left(\mathrm{f}_{\text {dis }}-\left(\mathrm{a}_{\mathrm{m}}^{H}\left(\mathrm{v}_{\mathrm{m}}^{H} / \mathrm{a}_{\mathrm{m}}^{H}\right)^{2} / 2+\mathrm{v}_{\mathrm{m}}^{H}\left(\Gamma-\mathrm{v}_{\mathrm{m}}^{H} / \mathrm{a}_{\mathrm{m}}^{H}\right)\right)\right) / \Gamma\right)\left(\Gamma>\mathrm{v}_{\mathrm{m}}^{H} / \mathrm{a}_{\mathrm{m}}^{H}\right)$, respectively. When (3) $\Gamma_{t m}>\mathrm{v}_{\mathrm{m}}^{H} / \mathrm{a}_{\mathrm{m}}^{H}, v_{\mathrm{m}}^{U}$ can be determined by $\mathrm{v}_{\mathrm{m}}^{U}=\min \left(v_{h o b}, v_{o p b}\right)$, where $v_{h o b}$ and $v_{o p b}$ represent, when $\Gamma_{t t t}>\mathrm{v}_{\mathrm{m}}^{H} / \mathrm{a}_{\mathrm{m}}^{H}$, the allowed maximum velocity of the dynamic deforming obstacles without collision with robots when they move at same direction and at opposite direction, respectively. $v_{h o b}$ and $v_{o p h}$ can be calculated by $v_{h o b}=\left(\mathrm{a}_{\mathrm{m}}^{H}\left(\mathrm{v}_{\mathrm{m}}^{H} / \mathrm{a}_{\mathrm{m}}^{H}\right)^{2} / 2+\mathrm{v}_{\mathrm{m}}^{H}\left(\Gamma_{t h t}-\mathrm{v}_{\mathrm{m}}^{H} / \mathrm{a}_{\mathrm{m}}^{H}\right)\right) \cos (\pi / 4) / \Gamma$ and $v_{\text {oph }}=\left(\mathrm{f}_{\mathrm{dis}}-\right.$ $\left.\left(\mathrm{a}_{\mathrm{m}}^{H}\left(\mathrm{v}_{\mathrm{m}}^{H} / \mathrm{a}_{\mathrm{m}}^{H}\right)^{2} / 2+\mathrm{v}_{\mathrm{m}}^{H}\left(\Gamma-\mathrm{v}_{\mathrm{m}}^{H} / \mathrm{a}_{\mathrm{m}}^{H}\right)\right)\right) / \Gamma$.

If the following barriers robots lost the sense to the target and the communication with others and lost, or the robots are damaged by the deforming obstacles when the robots are following barriers since the actual velocity of deforming obstacles are greater than the given speed in this section, and if the number of lost robots or damaged robots is less, since the number of swarm robots is more, it doesn't affect the stability of the hunting systems. This also reflects good robustness of the swarm robotic systems.

\subsection{Special Case 3}

There are several situations to analyze the stability of the hunting systems for the robots satisfying the conditions of following barriers when the barriers are robots. Theoretically, there are $m=\left\lfloor 2 \pi \mathrm{c}_{\mathrm{r}} / f_{\text {dis }}\right\rfloor$ robots, where $\lfloor\cdot\rfloor$ represents down rounding, on the effective hunting 
circle. That has the relationship with the condition $\left\|\boldsymbol{p}_{a j}\right\|>\mathrm{f}_{\text {dis }}$ and $\left\|\boldsymbol{p}_{a b}\right\|>2 \mathrm{f}_{\text {dis }}$ for robots to stop following barriers. But in fact, more robots, more coordinative difficulties to increase overall. There are less $m=\left\lfloor 2 \pi \mathrm{c}_{\mathrm{r}} / \mathrm{f}_{\text {dis }}\right\rfloor$ robots on the effective hunting circle since the difficulties increasing for robots from following barriers state into hunting state and to reach the effective hunting circle. When (1) $0<m \leq\left\lceil 2 \pi \mathrm{c}_{\mathrm{r}} /\left(2 \mathrm{f}_{\mathrm{dis}}\right)\right]$, where $\lceil\cdot\rceil$ represents up rounding, it is easy for all the robots to get to the effective hunting circle since when $0<m \leq\left\lfloor 2 \pi \mathrm{c}_{\mathrm{r}} /\left(2 \mathrm{f}_{\mathrm{dis}}\right)\right\rfloor$, there are enough space for one more robot to join in to get to the effective hunting circle.

When (2) $\left\lceil 2 \pi \mathrm{c}_{\mathrm{r}} /\left(2 \mathrm{f}_{\text {dis }}\right)\right]<m \leq\left\lfloor 2 \pi \mathrm{c}_{\mathrm{r}} / \mathrm{f}_{\text {dis }}\right\rfloor$, the more robots, the more difficulties from following barriers state into hunting state to get to the effective hunting circle. In addition to the special circumstances, all follow barriers robots will turn into hunting state so as to get to the effective hunting circle. In most cases, majority of the robots are on the effective hunting circle and small parts of the robots are following barriers out of the effective hunting circle, where "barriers" are robots. When (3) $m>\left\lfloor 2 \pi \mathrm{c}_{\mathrm{r}} / f_{\text {dis }}\right\rfloor$, there are some robots are on the effective hunting circle and some are always following barriers, where "barriers" are also robots. From the analyses of the above, we know that the systems can achieve hunting success for different $m$, and the swarm robotics systems are stable and have high scalability.

According to the Theorems 1 to 4, and above analyses, we can conclude that the whole swarm robotic systems for hunting are stable and have good scalability in an unknown complex environment.

\section{Simulations}

According to the model of hunting task in section 2.2, in this section, we will discuss swarm robots for hunting in both a no obstacles environment and an unknown complex environment with dynamic deforming obstacles. System parameters of the simulations are listed in Table 1. The avoiding collisions parameters of the hunting robots for different neighbors are listed in Table 2, and the avoiding collisions parameters of the target are listed in Table 3.

Table 1. The Hunting Systems Parameters

\begin{tabular}{cccccccccccccccccccccccc}
\hline Parameters & $\mathrm{c}_{1}$ & $\mathrm{c}_{2}$ & $\mathrm{~d}_{1}$ & $\mathrm{l}$ & $\mathrm{c}_{\mathrm{r}}$ & $\mathrm{p}_{\mathrm{r}}^{T}$ & $\mathrm{~s}_{\mathrm{r}}^{H}$ & $\mathrm{~s}_{\mathrm{r}}^{T}$ & $\rho_{h}$ & $\rho_{t}$ & $\varepsilon_{1}$ & $\varepsilon_{2}$ & $\mathrm{v}_{\omega}^{T}$ & $\mathrm{~V}_{\mathrm{m}}^{T}$ & $\mathrm{~V}_{\mathrm{m}}^{H}$ & $\mathrm{a}_{\mathrm{m}}^{H}$ & $\omega_{\mathrm{m}}^{H}$ & $\omega_{\mathrm{am}}^{H}$ \\
\hline Values & 0.8 & 3.2 & 5.1 & 12 & 9 & 6.4 & 50 & 10.5 & 3.5 & 8 & 0.02 & 0.6 & 0.25 & 0.9 & 0.9 & 40 & 8.0 & 180 \\
\hline
\end{tabular}

\subsection{Simulations and Analyses in a No Obstacles Environment}

In the first simulation, there is no obstacle considered in the environment. The initial position of swarm robots are shown in Table 4 that are given arbitrarily. In this simulation, the value of $\Gamma$ is 0.45 and is less than the lower limit 0.59 of $\Gamma$ computed according to the systems parameters listed in Table 1 and (27). However, the prey does not turn at each step, so it can also be hunted successfully. Since $\mathrm{f}_{\text {dis }}=2.5, \quad \mathrm{c}_{\mathrm{r}}=9,2 \pi \mathrm{c}_{\mathrm{r}} /\left(2 \mathrm{f}_{\text {dis }}\right)=11.3097, m=12=$ $\left\lceil 2 \pi \mathrm{c}_{\mathrm{r}} /\left(2 \mathrm{f}_{\text {dis }}\right)\right\rceil$. According to the stability analyses in section 4.7 , all the robots can reach the effective hunting circle and form a uniform distribution hunting formation.

5.1.1. Simulation Results: The hunting simulation trajectories of swarm robots according to SVF-model are shown in Figure 5, where a dotted-line circle represents the effective hunting circle, and the inter of the solid-line circle represents the potential domain of the prey. From 
Table 2. Avoiding Collisions Parameters of the Hunting Robots

\begin{tabular}{cccccccc}
\hline Parameters & $\mathrm{a}_{\text {dis }}^{1}$ & $\mathrm{a}_{\text {dis }}^{2}$ & $\mathrm{a}_{\text {dis }}^{3}$ & $\mathrm{~d}_{2}^{1}$ & $\mathrm{~d}_{2}^{2}$ & $\mathrm{~d}_{2}^{3}$ & $\mathrm{f}_{\text {dis }}$ \\
\hline Values & 1.1 & 1.8 & 3.5 & 1.2 & 2.2 & 2.2 & 2.5
\end{tabular}
Table 3. Avoiding Collisions
Parameters of the Target

\begin{tabular}{ccccccc}
\hline Parameters & $\mathrm{a}_{\mathrm{r}}^{S}$ & $\mathrm{a}_{\mathrm{r}}^{U}$ & $\mathrm{~d}_{3}$ & $\mathrm{~d}_{4}$ & $\rho_{s}$ & $\rho_{u}$ \\
\hline Values & 1.8 & 3.5 & 2.3 & 3.2 & 8.5 & 8.5 \\
\hline
\end{tabular}

Table 4. Initial Coordinate Values of Swarm Robots in Simulation 1

\begin{tabular}{ccccccccccccccccc}
\hline & $t_{1}$ & $h_{1}$ & $h_{2}$ & $h_{3}$ & $h_{4}$ & $h_{5}$ & $h_{6}$ & $h_{7}$ & $h_{8}$ & $h_{9}$ & $h_{10}$ & $h_{11}$ & $h_{12}$ \\
\hline $\mathrm{X}$ & -3.1 & 7.75 & -8.5 & -4.1 & 6.2 & 9.3 & -8.1 & -2.2 & -2.4 & -6.0 & -0.5 & -5.3 & -6.5 \\
\hline $\mathrm{Y}$ & 2.1 & -5.25 & -1.9 & -5.9 & -4.2 & -6.3 & -3.1 & -4.9 & -7.2 & -2.0 & -3.8 & -4.8 & -4.1 \\
\hline
\end{tabular}

Figure 5, similar to the hunting method based on LP-rule, the hunting algorithm based on SVF-model can emerge Leaders such as $h_{2}$ and $h_{4}$ in Figure 5(e), and the whole swarm emerge a uniform hunting formation in macro level finally. In Figs. 5(d) and 5(f), the movement direction changing of the prey is the results of its random selection.
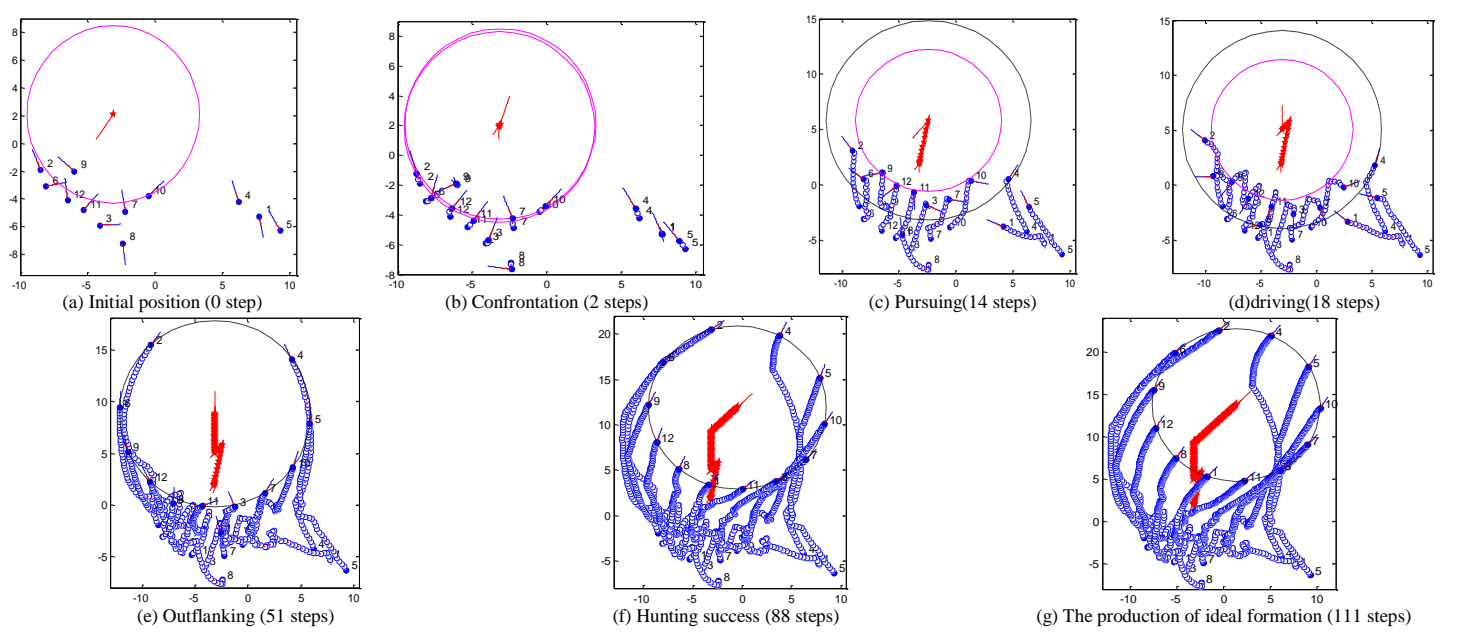

\section{Figure 5. Swarm Robots Self-organizing Hunting in a No Obstacle Environment}

5.1.2. Deviation Convergence Explanation: a) Analyses for the target distance deviation. In Figure 6, hunting robots move in and out of the effective hunting circle, which conforms to the actual situation. However, in [30], the hunting robots only move out of the convergence circle domain. Therefore, the convergence process in this paper is superior to that in [30].

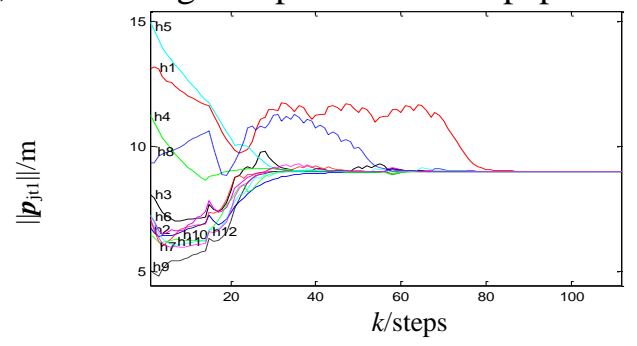

Figure 6. The Distance Deviation of Robots Toward Target 
b) Analyses for the resultant deviation of neighboring objects. We employ ${ }_{f_{a b}}$ to analyze the characteristics in the process of swarm robots achieving an ideal hunting formation. From Figure 7, we can see that, after about 101 steps, ${ }_{a b_{j}}$ of all individuals is close to zero. And the shock of Leaders $h_{2}$ and $h_{4}$ is minimal, which originally emerge in the process of outflanking. $h_{1}$ and $h_{11}$ have the most violent oscillation, which are the furthest relative to Leaders. This characteristic is consistent with that in [30]. Figure 7 shows that the conditions of robots d e c c i r e whether themselves are Leaders or not are deviation appearing approximate monotonic decay gradually after a larger value (This is opposite with the law in [30] about determining Leaders) such as $h_{2}$ and $h_{4}$ in Figure 7 . So the oscillation is small when Leaders are emerging.

\subsection{Simulation and Analyses for an Unknown Complex Environment with Dynamic Deforming Obstacles}

In this simulation, the environment includes four point static obstacles, a rotating cross, three static convex polygon obstacles, and three static non-convex obstacles. The scalability, robustness, flexibility, and performance of obstacle avoidance of the proposed algorithm will be tested in this section. The initial coordinates of the swarm robots are listed in Table 5. $\Gamma$ is 0.6 , which is bigger than the lower limit 0.59 obtained according to the systems parameters and (26). There is a cross rotating about a moving pivot. The rotational direction of the obstacle is counterclockwise, and the translational direction of the obstacle is the lower left. Its rotational angle velocity is 0.0462 , its translational linear velocity is 0.05 , and its radius is 6. Therefore, its biggest linear velocity is $0.3272 . v_{\mathrm{m}}^{u}=0.3350$, which can be calculated by section 4.6. Because $v_{\mathrm{m}}^{U}>0.3272$, as long as the following barriers robots can keep the update of the location of the target all the time and they can follow barriers successfully, and join in the hunting formation finally. The target will avoid obstacles when it meets static and/or dynamic obstacles. Since $m=8<\left\lceil 2 \pi \mathrm{c}_{\mathrm{r}} /\left(2 \mathrm{f}_{\text {dis }}\right)\right\rceil=12$, according to the stability analyses in section 4.7 , all the robots can reach the effective hunting circle and form the evenly distributed formation.
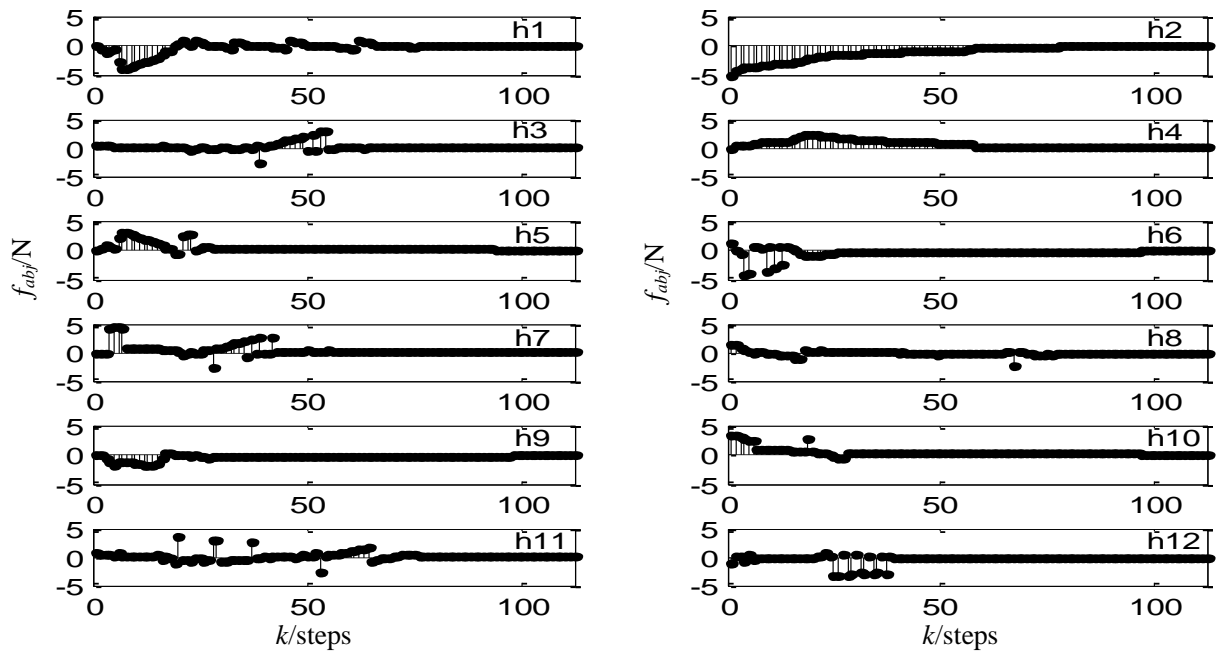

Figure 7. The Resultant Deviation of Neighboring Objects 
Table 5. Initial Coordinate Values of Swarm Robots in Simulation 2

\begin{tabular}{cccccccccc}
\hline & $t_{1}$ & $h_{1}$ & $h_{2}$ & $h_{3}$ & $h_{4}$ & $h_{5}$ & $h_{6}$ & $h_{7}$ & $h_{8}$ \\
\hline $\mathrm{X}$ & -3.1 & 3.5 & 10 & 0.5 & -5.5 & -5.4 & -7.5 & 2.1 & -2.1 \\
$\mathrm{Y}$ & 2.1 & -5.5 & -2.5 & -4.9 & -3.5 & -6.4 & -5.1 & -2.5 & -5.5 \\
\hline
\end{tabular}

Simulation trajectories are shown in Figure 8. In the simulation, the following barriers algorithm based on SVF-model successfully makes $h_{2}$ avoid the dynamic deforming obstacle, as shown in Figure 8(e). It can also be employed to avoid the static non-convex obstacles and convex obstacles successfully, as shown in Figure 8(h). For convex obstacles, non-convex obstacles, and dynamic convex obstacles easy to avoid, equation (4) is mainly used for obstacle avoidance.

The entire swarm robots can avoid obstacles and accomplish the hunting task smoothly with the number of robots decreasing 1/3 in an unknown complex environment. That reflects the algorithm not only has better scalability than the method based on LP-rule, but also has better performance of collision avoidance/obstacle avoidance, robustness, and flexibility, but
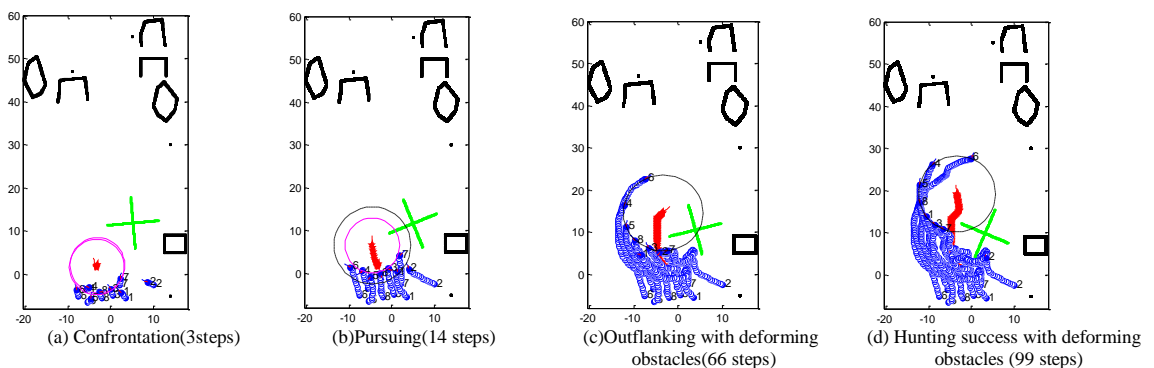

obstacles ( 99 steps)
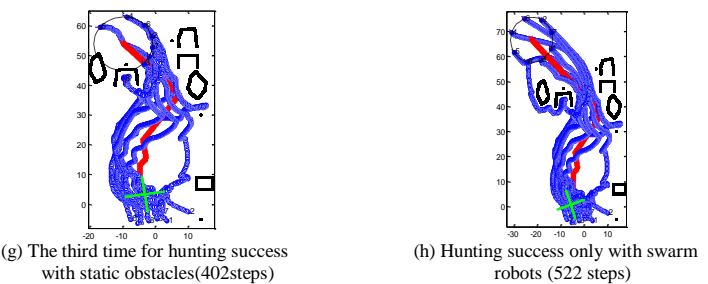

Hunting success with static
obstacles again(295 steps)

Figure 8. Swarm Robots Hunting in Unknown Cluttered Environments

LP - Rule doesn't consider how to make obstacle avoidance.

\section{The Comparative Analyses between SVF-Model and LP-Rule}

The advantages of the hunting algorithm based on SVF-model comparing with the method based on LP-rule in [30] are as follows:

\subsection{Simple Model}

In the SVF-Model, not only are calculation formulas simple, but also the physical meaning is clear. However, after determining the direction of loose space for 16 different hunting movement cases, a unified rule is established, i.e., LP-rule. 


\subsection{Hunting Success in Special Situations}

The special cases of two nearest neighbors on the direction to the target and so on are dealt with in SVF-model. For example, $h_{1}, h_{4}$, and $h_{5}$ are on the same direction to the target, as shown in Figure 6(a) . But those special cases aren't dealt with in LP-rule. Therefore, collisions maybe happen in some situations and the robots cannot encircle the target.

\subsection{No Theoretical Defects}

Even the number of robots are fewer, the robots can evenly distribute around the target in SVF-model. Whereas for LP-rule when the number of robots is three or four, there isn't evenly distributed hunting formation, and it has to do with LP-Rule.

\subsection{Good Capacity of Obstacle Avoidance}

From the simulations in section 5, the hunting algorithm based on SVF-model has the good capacity of avoiding static convex obstacles, non-convex obstacles, and dynamic deforming obstacles. Whereas there is no consideration how to make obstacle avoidance in LP-rule.

\subsection{Reasonable Speed Setting}

In LP-rule, when two nearest neighbors are all at the $S^{-}$or $S^{+}$, as shown in Figure 4 of [30], the further the distance from $h_{j}$ to the two nearest neighbors, the greater the speed of $h_{j}$ far away from the two nearest neighbors, and that makes the oscillation is larger when the Leaders emerge; the nearer the distance, the smaller the speed far away from the two nearest neighbors, and that makes hard for robots to avoid obstacles. However, the speed setting in SVF-model is precisely opposite to it in LP-rule. That is reason why the emerging laws of Leaders in SVF-model and LP-rule are just the opposite.

\subsection{Considering the Actual Physics Size of Robots}

The proposed algorithm considered the size of the actual robots constraints: the number of robots on the effective hunting circle, and that accords with the actual situations. However, there is no consideration about constraining the number of robots on the effective hunting circle in LP-rule.

\subsection{System Stability Theorems are Beneficial for the System Parameters Settings}

The sufficient conditions of system stability theorems are beneficial for system parameters settings. Thus the hunting processes are prone to oscillation of smaller and have more optimal trajectories. But the stability analysis for LP - rule has no guidance for system parameter settings.

\section{Conclusion}

In this paper, a hunting method based on a simplified virtual-force model is proposed for nonholonomic mobile swarm robots. The sufficient conditions for the system stability are given. Furthermore, the method based on SVF-model can make the group of robots emerge the expected collective behaviors, which is beneficial for the research of emergence control. Additionally, there is no local minimum problem for individuals' movement based on SVFmodel. Finally, the advantages of the proposed method compared with LP-rule are discussed. 


\section{Acknowledgements}

The authors are grateful to the anonymous reviewers and to the support of the National Natural Science Foundation of China (No. 61174140, No. 51374107), the Key Projects in the National Science and Technology Pillar Program (No. 2012BAH09B02), the National Natural Science Youth Foundation of China (No. 61203309), the Natural Science Foundation of Hunan (China) (No. 13JJ8014), the Education Department of Hunan Province Outstanding Youth Project (No. 12B043), and the Doctor Foundation (No. 20110161110035).

\section{References}

[1] M. Brambilla, E. Ferrante, M. Birattari, and M. Dorigo, "Swarm robotics: a review from the swarm engineering perspective", Swarm Intelligence, vol. 7, no. 1, (2013), pp. 1-41.

[2] T. A. Phan and R. A. Russell, "A swarm robot methodology for collaborative manipulation of non-identical objects", The International Journal of Robotics Research, vol. 31, no. 1, (2012), pp. 101-122.

[3] E. F. Parra-González and J. G. Ramírez-Torres, "Cooperative multi-robot box-pushing in a cluttered environment", Electronics, Robotics and Automotive Mechanics Conference, 2008. CERMA'08,(2008), pp. 514-519.

[4] K. Lerman, "A model of adaptation in collaborative multi-agent systems", Adaptive Behavior, vol. 12, no. 34, (2004), pp. 187-197.

[5] F. Mondada, L. M. Gambardella, D. Floreano, S. Nolfi, J.-L. Deneuborg, and M. Dorigo, "The cooperation of swarm-bots: Physical interactions in collective robotics", Robotics \& Automation Magazine, IEEE, vol. 12, no. 2, (2005), pp. 21-28.

[6] W. Liu and A. Winfield, "Modelling and optimisation of adaptive foraging in swarm robotic systems", The International Journal of Robotics Research, vol. 29, no. 14, (2010), pp. 1743-1760.

[7] A. H. Purnamadjaja and R. A. Russell, "Pheromone communication in a robot swarm: necrophoric bee behaviour and its replication", Robotica, vol. 23, no. 06, (2005), pp. 731-742.

[8] J. Wessnitzer and C. Melhuish, "Collective decision-making and behaviour transitions in distributed ad hoc wireless networks of mobile robots: Target-hunting", in Advances in Artificial Life, $1^{\text {st }}$ edtion, Springer Berlin Heidelberg (2003), vol.2801, pp. 893-902.

[9] J. P. Hespanha, H. J. Kim, and S. Sastry, "Multiple-agent probabilistic pursuit-evasion games", Decision and Control, 1999. Proceedings of the 38th IEEE Conference on, vol. 3,(1999), pp. 2432-2437.

[10] R. Vidal, O. Shakernia, H. J. Kim, D. H. Shim, and S. Sastry, "Probabilistic pursuit-evasion games: theory, implementation, and experimental evaluation", Robotics and Automation, IEEE Transactions on, vol. 18, no. 5, (2002), pp. 662-669.

[11] V. Isler, D. Sun, and S. Sastry, "Roadmap Based Pursuit-Evasion and Collision Avoidance", Robotics: Science and Systems, vol. 1,(2005), pp. 257-264.

[12] P. Chen and S. Sastry, "Pursuit controller performance guarantees for a lifeline pursuit-evasion game over a wireless sensor network", Decision and Control, 2006 45th IEEE Conference on,(2006), pp. 691-696.

[13] M. A. Vieira, R. Govindan, and G. S. Sukhatme, "Scalable and practical pursuit-evasion with networked robots", Intelligent Service Robotics, vol. 2, no. 4, (2009), pp. 247-263.

[14] S. D. Bopardikar, F. Bullo, and J. P. Hespanha, "On discrete-time pursuit-evasion games with sensing limitations", Robotics, IEEE Transactions on, vol. 24, no. 6, (2008), pp. 1429-1439.

[15] H. Yamaguchi, "A distributed motion coordination strategy for multiple nonholonomic mobile robots in cooperative hunting operations", Robotics and Autonomous Systems, vol. 43, no. 4, (2003), pp. 257-282.

[16] M. Wu, F. Huang, L. Wang, and J. Sun, "A distributed multi-robot cooperative hunting algorithm based on limit-cycle", Informatics in Control, Automation and Robotics, 2009. CAR'09. International Asia Conference on,(2009), pp. 156-160.

[17] C. Muro, R. Escobedo, L. Spector, and R. P. Coppinger, "Wolf-pack (Canis lupus) hunting strategies emerge from simple rules in computational simulations", Behavioural processes, vol. 88, no. 3, (2011), pp. 192-197.

[18] C. Ze-su, Z. Jie, and C. Jian, "Formation Control and Obstacle Avoidance for Multiple Robots Subject to Wheel-Slip", INTERNATIONAL JOURNAL OF ADVANCED ROBOTIC SYSTEMS, vol. 9, no. 188, (2012), pp. 1-15.

[19] Z. Cao, C. Zhou, L. Cheng, Y. Yang, W. Zhang, and M. Tan, "A Distributed Hunting Approach for Multiple Autonomous Robots", INTERNATIONAL JOURNAL OF ADVANCED ROBOTIC SYSTEMS, vol. 10, no. 2013, (2013), pp. 1-8. 
[20] U. Ruiz, R. Murrieta-Cid, and J. L. Marroquin, "Time-Optimal Motion Strategies for Capturing an Omnidirectional Evader Using a Differential Drive Robot", IEEE Transactions on Robotics,vol. 29, no. 5, (2013), pp. 1180-1196.

[21] Y. An, S. Li, and D. Lin, "Multiple Robotic Fish's Target Search and Cooperative Hunting Strategies", TELKOMNIKA Indonesian Journal of Electrical Engineering, vol. 12, no. 1, (2014), pp. 186-196.

[22] H. Sayyaadi and M. Sabet, "Nonlinear dynamics and control of a set of robots for hunting and coverage missions", International Journal of Dynamics and Control, no. 4, (2014), pp. 1-22.

[23] F. Liu and A. Narayanan, "Collision Avoidance and Swarm Robotic Group Formation", International Journal of Advanced Computer Science, vol. 4, no. 2, (2014), pp. 64-70.

[24] L. Schenato, S. Oh, S. Sastry, and P. Bose, "Swarm coordination for pursuit evasion games using sensor networks", Robotics and Automation, 2005. ICRA 2005. Proceedings of the 2005 IEEE International Conference on,(2005), pp. 2493-2498.

[25] X. Ju-Feng and T. Guan-Zheng, "Virtual Forces based approach for target capture with swarm robots", Control and Decision Conference, 2009. CCDC'09. Chinese,(2009), pp. 642-646.

[26] Z. Shi, X. Zhang, J. Tu, L. Liu, and J. Wei, "An improved capturing algorithm based on particle swarm optimization for swarm robots system", Computer Science and Automation Engineering (CSAE), 2012 IEEE International Conference on, vol. 2,(2012), pp. 566-571.

[27] L. Blazovics, K. Csorba, B. Forstner, and C. Hassan, "Target tracking and surrounding with swarm robots", Engineering of Computer Based Systems (ECBS), 2012 IEEE 19th International Conference and Workshops on,(2012), pp. 135-141.

[28] M. Kubo, H. Sato, A. Yamaguchi, and T. Yoshimura, "Target Enclosure for Multiple Targets", in Intelligent Autonomous Systems 12, ${ }^{\text {st }}$ edition, Springer Berlin Heidelberg (2013), vol.194, pp. 795-803.

[29] L. C. Pimenta, G. A. Pereira, N. Michael, R. C. Mesquita, M. M. Bosque, L. Chaimowicz, and V. Kumar, "Swarm coordination based on smoothed particle hydrodynamics technique", IEEE Transactions on Robotics, vol. 29, no. 2, (2013), pp. 383-399.

[30] T.-Y. HUANG, X.-B. CHEN, W.-B. XU, Z.-W. ZHOU, and Z.-Y. REN, "A Self-organizing Cooperative Hunting by Swarm Robotic Systems Based on Loose-preference Rule", Acta Automatica Sinica, vol. 39, no. 1, (2013), pp. 57-68.

[31] W. M. Spears and D. F. Gordon, "Using Artificial Physics to Control Agents", Information Intelligence and Systems, 1999. Proceedings. 1999 International Conference on,(1999), pp. 281-288.

[32] W.-b. Xu, X.-b. Chen, J. Zhao, and T.-y. Huang, "A Decentralized Method Using Artificial Moments for Multi-Robot Path-Planning", International Journal of Advanced Robotic Systems, vol. 10, no. 24, (2013), pp. $1-12$.

[33] J. D. Madden, R. C. Arkin, and D. R. MacNulty, "Multi-robot system based on model of wolf hunting behavior to emulate wolf and elk interactions", Robotics and Biomimetics (ROBIO), 2010 IEEE International Conference on,(2010), pp. 1043-1050.

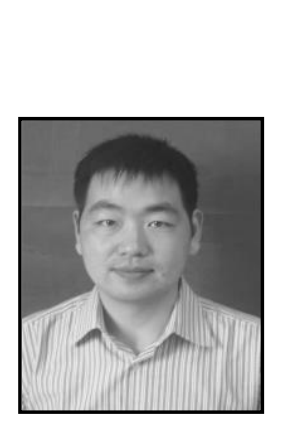

\begin{abstract}
Authors
Hongqiang Zhang received the B.S. and M.S. degrees in control science from Hunan University of Science and Technology in 2001 and Hunan University (HNU) in 2004, respectively. $\mathrm{He}$ is currently a $\mathrm{Ph}$. $\mathrm{D}$. candidate in control science and engineering in HNU. His research interests are swarm robotics system, swarm intelligence, optimization, and intelligent control.
\end{abstract}

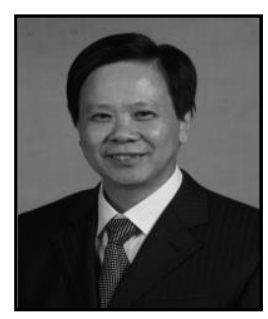

Jing Zhang received the B.S., M.S., and Ph.D. degree in control science from HNU in 1982, 1984, and 1997, respectively. He is currently a professor in the College of Electrical and Information Engineering, HNU. His research interests include networked control systems, optimization and intelligent control. 


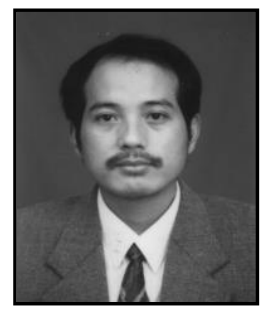

Shaowu Zhou received the B.S degree from ChongQing University in 1984,the M.S. degree from Central South Industry University in 199 0 , and the Ph.D.degree in Hunan University in 2005 . He is currently a $\mathrm{f}$ ull professor in HunanUniversity of Science and Technology. His resea rch interests are nonlinear system control and robust control.

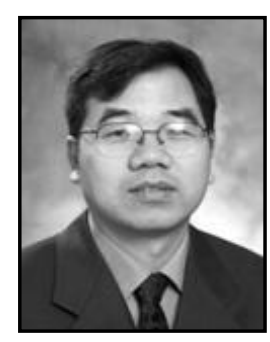

Puren Ouyang is an associate professor in the Department of Aerospace Engineering at Ryerson University, Canada. $\mathrm{He}$ is also a special professor at Hunan University of Science and Technology, China. He has been working in the areas of robotics and control, Mechatronics, and precision manipulator and devices. Dr. Ouyang has also been specialized in robotic system with compliant mechanisms.

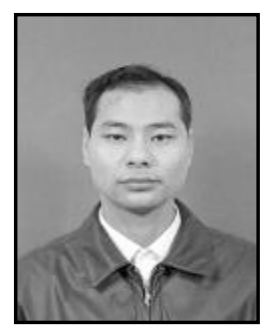

LianghongWu received the B.S. degree in industrial automation fro mHunan University of Science and Technology in 2001, and the M.S. a nd $\mathrm{Ph} . \mathrm{D}$. degree in control science and engineering from Hunan Univer sity in 2007 and 2011, respectively. He is currently an associate profess or in Hunan University of Science and Technology. His research intere sts includeartificial intelligent, evolutionary computation and applicatio ns. 
International Journal of Control and Automation Vol.8, No.11 (2015) 